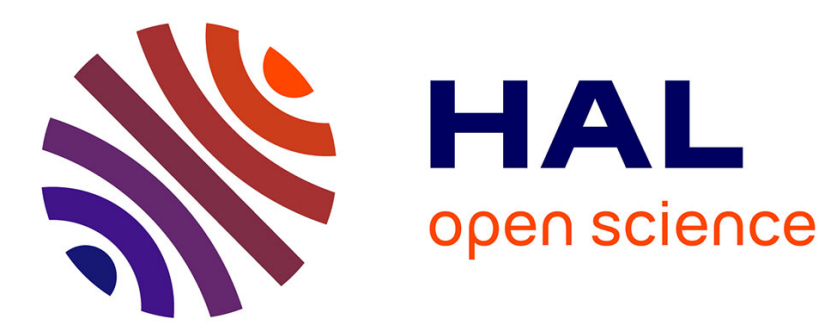

\title{
Trace Theorems for a Class of Ramified Domains with Self-Similar Fractal Boundaries
}

\author{
Yves Achdou, Nicoletta Tchou
}

\section{To cite this version:}

Yves Achdou, Nicoletta Tchou. Trace Theorems for a Class of Ramified Domains with SelfSimilar Fractal Boundaries. SIAM Journal on Mathematical Analysis, 2010, 42 (4), pp.1449-1482. 10.1137/090747294. hal-00353135v2

\section{HAL Id: hal-00353135 \\ https://hal.science/hal-00353135v2}

Submitted on 10 Dec 2009

HAL is a multi-disciplinary open access archive for the deposit and dissemination of scientific research documents, whether they are published or not. The documents may come from teaching and research institutions in France or abroad, or from public or private research centers.
L'archive ouverte pluridisciplinaire HAL, est destinée au dépôt et à la diffusion de documents scientifiques de niveau recherche, publiés ou non, émanant des établissements d'enseignement et de recherche français ou étrangers, des laboratoires publics ou privés. 


\title{
Trace Theorems for a Class of Ramified Domains with Self-Similar Fractal Boundaries
}

\author{
Yves Achdou ${ }^{*}$ Nicoletta Tchou †
}

November 29, 2009

\begin{abstract}
This work deals with trace theorems for a class of ramified bidimensional domains $\Omega$ with a self-similar fractal boundary $\Gamma^{\infty}$. The fractal boundary $\Gamma^{\infty}$ is supplied with a probability measure $\mu$ called the self-similar measure. Emphasis is put on the case when the domain is not a $\epsilon-\delta$ domain as defined by Jones and the fractal set is not totally disconnected. In this case, the classical trace results cannot be used. Here, the Lipschitz spaces with jumps recently introduced by Jonsson play a crucial role. Indeed, it is proved in particular that if the Hausdorff dimension $d$ of $\Gamma^{\infty}$ is not smaller than one, then the space of the traces of functions in $W^{m+1, q}(\Omega), m \in \mathbb{N}, 1<q<\infty$ is $\operatorname{JLip}\left(m+1-\frac{2-d}{q}, q, q ; m ; \Gamma^{\infty}\right)$. The proof is elementary; a main step is a strengthened trace inequality in the norm $L_{\mu}^{q}\left(\Gamma^{\infty}\right)$.
\end{abstract}

\section{Introduction}

This work deals with some properties of the Sobolev spaces $W^{m+1, q}(\Omega), m \in \mathbb{N}$, for a class of ramified domains $\Omega$ of $\mathbb{R}^{2}$ with a self-similar fractal boundary called $\Gamma^{\infty}$ below, see for example Figures 1 and 3. The domain $\Omega$ essentially depends on a parameter $a, 0<a \leq a^{*}$. As explained below, the restriction $a \leq a^{*}$ allows for the construction of $\Omega$ as a union of non-overlapping sub-domains, see (10) below.

Such a geometry can be seen as a bidimensional idealization of the bronchial tree, for example. Indeed, the present work is a continuation of [1] and of [2], which were part of a wider project aimed at simulating the diffusion of medical sprays in lungs. Since the exchanges between the lungs and the circulatory system take place only in the last generations of the bronchial tree (the smallest structures), reasonable models for the diffusion of, e.g., oxygen may involve a non-homogeneous Neumann or Robin condition on the boundary $\Gamma^{\infty}$. Similarly, the lungs are mechanically coupled to the diaphragm, which also implies non-homogeneous boundary conditions on $\Gamma^{\infty}$, if one is interested in a coupled fluid-structure model. It is therefore necessary to study traces of functions on $\Gamma^{\infty}$; here, we will focus on functions belonging to $W^{m+1, q}(\Omega)$, for $m \in \mathbb{N}$ and $1<q<\infty$.

Function spaces defined in irregular domains have been widely studied in the literature:

- Jones [8] (and Vodopjanov et al [25] in the case $n=2$, see also [17, 11]) have studied the open bounded subsets $\Omega$ of $\mathbb{R}^{n}$ such that there exists a continuous extension operator from $W^{\ell, p}(\Omega)$ in $W^{\ell, p}\left(\mathbb{R}^{n}\right)$, for all nonnegative integers $\ell$ and real numbers $p, 1 \leq p \leq \infty$.

*UFR Mathématiques, Université Paris Diderot, Case 7012, 175 rue du Chevaleret, 75013 Paris, France and UMR 7598, Laboratoire Jacques-Louis Lions, F-75005, Paris, France. achdou@math.jussieu.fr

${ }^{\dagger}$ IRMAR, Université de Rennes 1, Rennes, France, nicoletta.tchou@univ-rennes1.fr 
Jones has proved that if $\Omega$ is a $\epsilon-\delta$ domain for some parameters $\epsilon, \delta>0$, see $[8,11]$ for the definition, then the above extension property is true. Moreover, in dimension two, if the extension property stated above is true, then $\Omega$ is a $\epsilon-\delta$ domain for some parameters $\epsilon, \delta>0$. In dimension two, the definition of such domains is equivalent to that of quasi-disks, see [17].

- Jonsson and Wallin [11] have considered closed subsets $F$ of $\mathbb{R}^{n}$ supplied with a Borel measure $\mu$ such that there exists a positive real number $d$ and two positive constants $c_{1}$ and $c_{2}$ with

$$
c_{1} r^{d} \leq \mu(B(x, r)) \leq c_{2} r^{d}
$$

for all $x \in F$ and $r<1$ (here $B(x, r)$ is the ball in $F$ with center $x$ and radius $r$, with respect to the Euclidean distance in $\mathbb{R}^{n}$ ); in [11], these sets are called $d$-sets.

Concerning the set $\Gamma^{\infty}$ studied in the present paper, we will recall in $\S 3.2$ below the notion of self-similar measure $\mu$ defined in the triplet $\left(\Gamma^{\infty}, F_{1}, F_{2}\right)$, see [13]. With the Borel regular probability measure $\mu, \Gamma^{\infty}$ is a $d$-set where $d$ is the Hausdorff dimension of $\Gamma^{\infty}$.

In [11], Sobolev and Besov spaces are defined on the $d$-sets, and extension and trace results for Besov and Sobolev spaces are proved using as a main ingredient Whitney extension theory. In particular, see [11] page 182, there exists a continuous trace operator from $W^{1, p}\left(\mathbb{R}^{n}\right)$ onto $B_{1-\frac{n-d}{p}}^{p, p}(F)$, if $\max (1, n-d)<p<\infty$, where, for $0<s<1$,

$$
B_{s}^{p, p}(F)=\left\{f \in L_{\mu}^{p}(F) ; \int_{x, y \in F,|x-y|<1} \frac{|f(x)-f(y)|^{p}}{|x-y|^{d+s p}} d \mu(x) d \mu(y)<\infty\right\},
$$

see the definition in [11] page 103. A more general trace theorem is available, see Theorem 1 , page 141 in [11].

The approach of Triebel [24] is somewhat different. In [24] chapter IV, paragraph 18, it is proved that the space of the traces of functions in $B_{\frac{n-d}{p}}^{p, q}\left(\mathbb{R}^{n}\right)$ is $L_{\mu}^{p}(F)$ for $0<d<n$, $d / n<p<\infty$ and $0<q \leq \min (1, p)$; Besov spaces on $F$ are then defined as the space of the traces of Besov spaces on $\mathbb{R}^{n}$ and embeddings properties are studied.

- There is also a growing interest in analysis on self-similar fractal sets, see for instance Kigami [13], Strichartz [22, 23], Mosco[20, 19] and references therein. These works aim at intrinsically defining function spaces using Dirichlet forms and a different metric from the Euclidean one. The results in this direction are often subject to the important assumption that the set is post-critically finite (or p.c.f.), see [13], page 23 for the definition. In a different direction, Jonsson has studied Lipschitz functions spaces on self-similar fractal sets under a technical condition which yields a Markov inequality at any order, see the pioneering works $[9,10]$. This theory does not require the fractal set to be post-critically finite. In [10], Lipschitz functions spaces allowing jumps at some special points in the self-similar fractal set have been introduced, along with Haar wavelets of arbitrary order. The previously mentioned function spaces can be characterized using the coefficients of the expansion in some high order Haar wavelet bases. The theory in [10] is the cornerstone of the present paper. It will be briefly reviewed in $\S 4$.

- The question of extensions or traces naturally arises in boundary value or transmission problems in domains with fractal boundaries. Results in this direction have been given in $[21,15,14]$ for the Koch flake. Here also, the assumption that the fractal set is p.c.f. is generally made. 
Our goal here is to study the traces of functions of $W^{m+1, q}(\Omega)$ on the fractal boundary $\Gamma^{\infty}$. Note that this is different from considering the traces of functions of $W^{m+1, q}\left(\mathbb{R}^{2}\right)$ on $\Gamma^{\infty}$.

If $a<a^{*}$, then $\Omega$ is a $\epsilon-\delta$ domain. In this case, the results of Jones [8] and those of Jonsson and Wallin [11] can be combined to obtain trace results. This has been done in [2]: if $\max (1,2-d)<$ $q<\infty$, the space of the traces of functions in $W^{1, q}(\Omega)$ is $B_{1-(2-d) / q}^{q, q}\left(\Gamma^{\infty}\right)$, where $d$ is the Hausdorff dimension of $\Gamma^{\infty}$.

When $a=a^{*}$, the situation is more complicated because

- $\Omega$ is not a $\epsilon-\delta$ domain,

- $\Gamma^{\infty}$ may not be totally disconnected. It may even be non post-critically finite.

In [2] we mainly focused on the case $a=a^{*}$, and especially on the geometry presented in $\S 2.2 .2$ below, see Figure 3. We studied some properties of the traces of functions in $H^{1}(\Omega)$, without completely characterizing the trace space though. The results proved in [2] are :

- if $a \leq a^{*}$ then the trace of a function in $H^{1}(\Omega)$ belongs to $L_{\mu}^{p}\left(\Gamma^{\infty}\right)$, for all real numbers $p$ such that $1 \leq p<\infty$.

- in the case $a=a^{*}$, we gave an example of a function in $H^{1}(\Omega)$ whose trace on $\Gamma^{\infty}$ has not a bounded mean oscillation with respect to $\mu$, (thus does not belong to $L_{\mu}^{\infty}\left(\Gamma^{\infty}\right)$ ).

- for the geometry displayed in Figure 3 in the critical case $a=a^{*}$, the trace of a function in $H^{1}(\Omega)$ belongs to the Besov space $B_{s}^{2,2}\left(\Gamma^{\infty}, \mu\right)$ for all real numbers $s$ such that $0 \leq s<d / 4$, where $d$ is the Hausdorff dimension of $\Gamma^{\infty}$, and there exists a function in $H^{1}(\Omega)$ whose trace does not belong to $B_{s}^{2,2}\left(\Gamma^{\infty}, \mu\right)$ for all $s>d / 4$. Note the important contrast with the case $a<a^{*}$ for which the trace of a function in $H^{1}(\Omega)$ belongs to $B_{s}^{2,2}\left(\Gamma^{\infty}, \mu\right)$ for $0 \leq s \leq d / 2$.

In the present paper, we aim at charaterizing the traces of functions in $W^{m+1, q}(\Omega)$ by using the results contained in [9] and mostly [10].

The article is organized as follows: the geometry is presented in Section 2. In Section 3, we recall some of the results of [1] on the space $W^{1, q}(\Omega)$, concerning Poincaré inequality and the construction of the trace operator. The theory proposed in [10] is reviewed in $\S 4$ where we mainly focus on the spaces $\operatorname{Jip}\left(s, q, q ; m ; \Gamma^{\infty}\right), m<s<m+1,1 \leq q<\infty$, that we will use later on. For simplicity, we first investigate the traces of functions in $W^{1, q}(\Omega)$ : the main result of the paper is Theorem 9 stated and proved in Section 5. The proof uses elementary ingredients, mainly the strengthened trace inequality stated in Theorem 11 . The traces of functions in $W^{m+1, q}(\Omega)$ for a positive integer $m$ are characterized in Section 6 .

The results presented here can be generalized to functions in $W^{s, q}(\Omega)$ for $s$ non integer or in Besov spaces: this topic is currently under investigation.

\section{The Geometry}

We first describe the general geometrical setting, then we give two relevant examples. 


\subsection{General Setting}

\subsubsection{The similitudes $F_{1}$ and $F_{2}$ and the self-similar set $\Gamma^{\infty}$}

Consider four real numbers $a, \alpha, \beta, \theta$ such that $0<a<1 / \sqrt{2}, \alpha>0, \beta>0$ and $0 \leq \theta<\pi / 2$. Let $F_{i}, i=1,2$ be the two similitudes in $\mathbb{R}^{2}$ given by

$$
\begin{aligned}
& F_{1}\left(\begin{array}{l}
x_{1} \\
x_{2}
\end{array}\right)=\left(\begin{array}{c}
-\alpha \\
\beta
\end{array}\right)+a\left(\begin{array}{c}
x_{1} \cos \theta-x_{2} \sin \theta \\
x_{1} \sin \theta+x_{2} \cos \theta
\end{array}\right), \\
& F_{2}\left(\begin{array}{l}
x_{1} \\
x_{2}
\end{array}\right)=\left(\begin{array}{c}
\alpha \\
\beta
\end{array}\right)+a\left(\begin{array}{c}
x_{1} \cos \theta+x_{2} \sin \theta \\
-x_{1} \sin \theta+x_{2} \cos \theta
\end{array}\right) .
\end{aligned}
$$

The two similitudes have the same dilation ratio $a$ and opposite angles $\pm \theta$. One can obtain $F_{2}$ by composing $F_{1}$ with the symmetry with respect to the axis $\left\{x_{1}=0\right\}$.

We call $\Gamma^{\infty}$ the self-similar set associated to the similitudes $F_{1}$ and $F_{2}$, i.e. the unique compact subset of $\mathbb{R}^{2}$ such that

$$
\Gamma^{\infty}=F_{1}\left(\Gamma^{\infty}\right) \cup F_{2}\left(\Gamma^{\infty}\right) .
$$

For $n \geq 1$, we call $\mathcal{A}_{n}$ the set containing all the $2^{n}$ mappings from $\{1, \ldots, n\}$ to $\{1,2\}$. We define

$$
\mathcal{M}_{\sigma}=F_{\sigma(1)} \circ \cdots \circ F_{\sigma(n)} \quad \text { for } \sigma \in \mathcal{A}_{n} .
$$

The definition of $\Gamma^{\infty}$ implies that for all $n>0$,

$$
\Gamma^{\infty}=\bigcup_{\sigma \in \mathcal{A}_{n}} \mathcal{M}_{\sigma}\left(\Gamma^{\infty}\right)
$$

We state without proof the following proposition, which says that, up to an affine map, the shape of $\Gamma^{\infty}$ does not depend on $\alpha$ and $\beta$.

Proposition 1 Let $F_{1}$ and $F_{2}$ be defined by (1) where the parameters a, $\theta, \alpha, \beta$ satisfy the assumptions above. Let the similitudes $G_{1}$ and $G_{2}$ be defined by

$$
G_{1}=\left(\begin{array}{c}
-\gamma \\
\delta
\end{array}\right)+F_{1}, \quad G_{2}=\left(\begin{array}{l}
\gamma \\
\delta
\end{array}\right)+F_{2}
$$

where $\alpha+\gamma>0$ and $\beta+\delta>0$. The self-similar set associated to $G_{1}$ and $G_{2}$ is

$$
\left(\frac{\alpha \delta}{\beta-\beta \gamma} \frac{0}{\beta a \sin \theta+\alpha(1-a \cos \theta)}\right)+\left(1+\frac{\delta a \sin \theta+\gamma(1-a \cos \theta)}{\beta a \sin \theta+\alpha(1-a \cos \theta)}\right) \Gamma^{\infty},
$$

where $\Gamma^{\infty}$ is the self-similar set associated to the similitudes $F_{1}$ and $F_{2}$.

Note that the assumptions in Proposition 1 imply that the parameter $\left(1+\frac{\delta a \sin \theta+\gamma(1-a \cos \theta)}{\beta a \sin \theta+\alpha(1-a \cos \theta)}\right)$ is positive.

The following theorem was stated by Mandelbrodt et al, [16]:

Theorem 1 For any $\theta, 0 \leq \theta<\pi / 2$, there exists a unique positive number $a^{*}<1 / \sqrt{2}$, (which depends on $\theta$ but not on $(\alpha, \beta)$ from Proposition 1) such that

$$
\begin{array}{clcc}
0<a<a^{*} & \Rightarrow \quad F_{1}\left(\Gamma^{\infty}\right) \cap F_{2}\left(\Gamma^{\infty}\right)=\emptyset & \Rightarrow & \Gamma^{\infty} \text { is totally disconnected, } \\
a=a^{*} & \Rightarrow \quad F_{1}\left(\Gamma^{\infty}\right) \cap F_{2}\left(\Gamma^{\infty}\right) \neq \emptyset & \Rightarrow & \Gamma^{\infty} \text { is connected, (from Th. 1.6.2 in [13]). }
\end{array}
$$


If $\theta>0$, then the critical parameter $a^{*}$ is the unique positive root of the polynomial equation:

$$
\sum_{i=0}^{\kappa-1} X^{i+2} \cos i \theta=\frac{1}{2}
$$

where $\kappa$ is the smallest integer such that $\kappa \theta \geq \pi / 2$. If $\theta=0$ then $a^{*}=1 / 2$.

Remark 1 From (4), it can be seen that $\theta \rightarrow a^{*}(\theta)$ is a continuous and increasing function from $[0, \pi / 2)$ onto $[1 / 2,1 / \sqrt{2})$.

Hereafter, for a given $\theta, 0 \leq \theta<\pi / 2$, we will only consider $a$ such that $0<a \leq a^{*}$.

\subsubsection{The simplest possible construction with $F_{1}$ and $F_{2}$}

Call $P_{1}=(-1,0)$ and $P_{2}=(1,0)$ and $\Gamma^{0}$ the line segment $\Gamma^{0}=\left[P_{1}, P_{2}\right]$. We impose that $F_{2}\left(P_{1}\right)$, and $F_{2}\left(P_{2}\right)$ have positive coordinates, i.e. that

$$
a \cos \theta<\alpha \text { and } a \sin \theta<\beta .
$$

We also impose that the open domain $\widetilde{Y}^{0}$ inside the closed polygonal line joining the points $P_{1}$, $P_{2}, F_{2}\left(P_{2}\right), F_{2}\left(P_{1}\right), F_{1}\left(P_{2}\right), F_{1}\left(P_{1}\right), P_{1}$ in this order is convex. With (5), this is true if and only if

$$
(\alpha-1) \sin \theta+\beta \cos \theta \geq 0 .
$$

Under assumptions (5) and (6), the domain $\widetilde{Y}^{0}$ is either hexagonal or trapezoidal in the degenerate cases (in particular if $\theta=0$ ), contained in the half-plane $x_{2}>0$ and symmetric w.r.t. the vertical axis $x_{1}=0$.

We first describe the simplest possible ramified domain containing the images of $P_{1}$ and $P_{2}$ by all the mappings $F_{i_{1}} \circ \cdots \circ F_{i_{n}}, i_{j}=1,2$. This domain will be noted $\widetilde{\Omega}$. The construction is as follows: it it possible to glue together $\overline{\widetilde{Y}^{0}}, F_{1}\left(\overline{\widetilde{Y}^{0}}\right)$ and $F_{2}\left(\overline{\widetilde{Y}^{0}}\right)$ and obtain a new polygonal domain, also symmetric with respect to the axis $\left\{x_{1}=0\right\}$. The assumptions (5) and (6) imply that $\widetilde{Y}^{0} \cap F_{1}\left(\widetilde{Y}^{0}\right)=\emptyset$ and $\widetilde{Y}^{0} \cap F_{2}\left(\widetilde{Y}^{0}\right)=\emptyset$. We also define the ramified open domain $\widetilde{\Omega}$, see Figures 1 and 3:

$$
\widetilde{\Omega}=\operatorname{Interior}\left(\overline{\widetilde{Y}^{0}} \cup\left(\bigcup_{n=1}^{\infty} \underset{\sigma \in \mathcal{A}_{n}}{\cup} \mathcal{M}_{\sigma}\left(\overline{\widetilde{Y}^{0}}\right)\right)\right) .
$$

Note that $\widetilde{\Omega}$ is symmetric with respect to the axis $x_{1}=0$, and that for $a<1 / \sqrt{2}$, the measure of $\widetilde{\Omega}$ is finite.

With $a^{*}$ defined as above, we shall make the following assumption on $\alpha$ and $\beta$ :

Assumption 1 For $0<\theta<\pi / 2$, the parameters $\alpha$ and $\beta$ satisfy (6) and (5) for $a=a^{*}$, and are such that

1. for all $a, 0<a \leq a^{*}$, the sets $\widetilde{Y}^{0}, \mathcal{M}_{\sigma}\left(\widetilde{Y}^{0}\right), \sigma \in \mathcal{A}_{n}, n>0$ are disjoint,

2. for all $a, 0<a<a^{*}, F_{1}(\overline{\widetilde{\Omega}}) \cap F_{2}(\overline{\widetilde{\Omega}})=\emptyset$,

3. for $a=a^{*}, F_{1}(\overline{\widetilde{\Omega}}) \cap F_{2}(\overline{\widetilde{\Omega}}) \neq \emptyset$.

In the case when $\theta=0$, Assumption 1 is satisfied by any $\alpha>a^{*}=1 / 2$ and $\beta>0$. The following theorem asserts that for any $\theta, 0<\theta<\pi / 2$, there exist $(\alpha, \beta)$ satisfying Assumption 1. 
Theorem 2 If $\theta \in(0, \pi / 2)$, then for all $\alpha>a^{*} \cos \theta$, there exists $\bar{\beta}>0$ such that $\bar{\beta}>a^{*} \sin \theta$ and $(\alpha-1) \sin \theta+\bar{\beta} \cos \theta \geq 0$ and for all $\beta \geq \bar{\beta},(\alpha, \beta)$ satisfies Assumption 1.

Proof. The proof of this result is based on elementary but tricky geometric considerations. We sketch it in Appendix A.

The following theorem gives different and explicit sufficient conditions for Assumption 1 to hold in the case when $\pi / 6 \leq \theta \leq \pi / 3$.

Theorem 3 1. If $\theta \in[\pi / 6, \pi / 4]$, then the pairs $(\alpha, \beta)$ satisfying (5) for $a=a^{*}$ and

$$
\beta \cos \theta>\max (1-\alpha, \alpha) \sin \theta
$$

satisfy Assumption 1.

2. If $\theta \in[\pi / 4, \pi / 3]$, then the pairs $(\alpha, \beta)$ satisfying (5) for $a=a^{*}$, (6) and

$$
\left\{\begin{aligned}
2 \cos \theta(\beta \sin \theta-\alpha \cos \theta) & >-\cos 2 \theta \\
(1-\alpha) \cos 3 \theta+\beta \sin 3 \theta & >0
\end{aligned}\right.
$$

satisfy Assumption 1.

Proof. See Appendix A.

Remark 2 We have observed numerically that for any $\theta, 0<\theta<\pi / 6$, the pairs $(\alpha, \beta)$ satisfying (5) for $a=a^{*}$ and (8) do satisfy Assumption 1, but we did not obtain a reasonably short proof.

Remark 3 Note that for $\theta=\pi / 4$, conditions (9) and (8) are equivalent.

For a given $\theta$, let $(\alpha, \beta)$ satisfy Assumption 1; for $0 \leq a<a^{*}$, the Moran condition (open set condition) see $[18,13]$ is satisfied (the Moran condition is that there exists a nonempty bounded open subset $O$ of $\mathbb{R}^{2}$ such that $F_{1}(O) \cap F_{2}(O)=\emptyset$ and $\left.F_{1}(O) \cup F_{2}(O) \subset O\right)$. Indeed, one can take $O=\widetilde{\Omega}$. From this, the Hausdorff dimension of $\Gamma^{\infty}$ is

$$
\operatorname{dim}_{H}\left(\Gamma^{\infty}\right)=d \equiv-\log 2 / \log a,
$$

see $[18,13]$. The assumption $a<1 / \sqrt{2}$ implies that $d<2$.

\subsubsection{More general constructions}

Let $(\alpha, \beta)$ satisfy Assumption 1. We assume that for all $a, 0<a \leq a^{*}$, (where $a^{*}$ has been introduced above), the polygonal cell $Y^{0}$ satisfies the following assumptions:

1. $Y^{0}$ is contained in the half-plane $x_{2}>0$.

2. $\partial Y^{0} \cap\left\{x_{2}=0\right\}=\Gamma^{0}=\left[P_{1}, P_{2}\right]$.

3. $F_{1}\left(\Gamma^{0}\right)$ and $F_{2}\left(\Gamma^{0}\right)$ are contained in some sides of $\partial Y^{0}$.

4. the sets $Y^{0}, \mathcal{M}_{\sigma}\left(Y^{0}\right), \sigma \in \mathcal{A}_{n}, n>0$ are disjoint.

With these assumptions, we can construct the ramified open domain

$$
\Omega=\operatorname{Interior}\left(\overline{Y^{0}} \cup\left(\bigcup_{n=1}^{\infty} \underset{\sigma \in \mathcal{A}_{n}}{\cup} \mathcal{M}_{\sigma}\left(\overline{Y^{0}}\right)\right)\right),
$$

see Figure 2 for an example. The self-similar fractal set $\Gamma^{\infty}$ is a subset of $\partial \Omega$. We split the boundary of $\Omega$ into $\Gamma^{\infty}, \Gamma^{0}=[-1,1] \times\{0\}$ and $\Sigma=\partial \Omega \backslash\left(\Gamma^{0} \cup \Gamma^{\infty}\right)$.

Remark 4 A further generalization is possible: all the assumptions above are maintained except that $\partial Y^{0} \backslash\left(\Gamma^{0} \cup F_{1}\left(\Gamma^{0}\right) \cup F_{2}\left(\Gamma^{0}\right)\right)$ may be made of curved lines. All what follows is valid in this case. For simplicity, we will still assume that $Y^{0}$ is polygonal. 


\subsubsection{Additional notations}

For what follows, it is important to define the polygonal open domain $Y^{N}$ obtained by stopping the above construction at step $N+1$,

$$
Y^{N}=\operatorname{Interior}\left(\overline{Y^{0}} \cup\left(\bigcup_{n=1}^{N} \underset{\sigma \in \mathcal{A}_{n}}{\cup} \mathcal{M}_{\sigma}\left(\overline{Y^{0}}\right)\right)\right) \text {. }
$$

We introduce the open domains $\Omega^{\sigma}=\mathcal{M}_{\sigma}(\Omega)$ and $\Omega^{N}=\cup_{\sigma \in \mathcal{A}_{N}} \Omega^{\sigma}=\Omega \backslash \overline{Y^{N-1}}$, for $N>0$. When needed, we will agree to say that $\Omega^{0}=\Omega$. We define the sets $\Gamma^{\sigma}=\mathcal{M}_{\sigma}\left(\Gamma^{0}\right)$ and $\Gamma^{N}=\cup_{\sigma \in \mathcal{A}_{N}} \Gamma^{\sigma}$. The one-dimensional Lebesgue measure of $\Gamma^{\sigma}$ for $\sigma \in \mathcal{A}_{N}$ and of $\Gamma^{N}$ are

$$
\left|\Gamma^{\sigma}\right|=a^{N}\left|\Gamma^{0}\right| \quad \text { and } \quad\left|\Gamma^{N}\right|=(2 a)^{N}\left|\Gamma^{0}\right| \text {. }
$$

\subsection{Two Examples}

\subsubsection{Example 1}

Let $F_{1}$ and $F_{2}$ be the affine maps in $\mathbb{R}^{2}$

$$
F_{1}(x)=\left(-\alpha+a x_{1}, \beta+a x_{2}\right), \quad F_{2}(x)=\left(\alpha+a x_{1}, \beta+a x_{2}\right),
$$

so $\theta=0$, and (5) becomes $a<\alpha$ and $\beta>0$. The domain $\tilde{Y}^{0}$ is trapezoidal:

$$
\widetilde{Y}^{0}=\operatorname{Interior}\left(\operatorname{convex} \operatorname{hull}\left(P_{1}, P_{2}, F_{2}\left(P_{2}\right), F_{1}\left(P_{1}\right)\right)\right) \text {. }
$$

The sets $\overline{\widetilde{Y}^{0}}, \mathcal{M}_{\sigma}\left(\overline{\widetilde{Y}^{0}}\right), \sigma \in \mathcal{A}_{n}, n>0$ do not overlap if $a \leq a^{*}=1 / 2$. The domain $\widetilde{\Omega}$ described in $\S 2.1 .2$ is shown in Figure 1 for $\alpha=3 / 2, \beta=3$ and $a=a^{*}=1 / 2$.

If $a<1 / 2$, the Hausdorff dimension of $\Gamma^{\infty}$ is smaller than 1 ; the set $\Gamma^{\infty}$ is a Cantor set, totally disconnected, (i.e. $F_{1}\left(\Gamma^{\infty}\right) \cap F_{2}\left(\Gamma^{\infty}\right)$ is empty), and contained in a straight line; it can be proved that $\widetilde{\Omega}$ is a $\epsilon-\delta$ domain as defined by Jones [8], see also [11] and [17].

In the critical case when $a=a^{*}=1 / 2, \Gamma^{\infty}$ is the straight line segment $[-2 \alpha, 2 \alpha] \times\{2 \beta\}$, so it is connected and its Hausdorff dimension is one. It is post-critically finite because $F_{1}\left(\Gamma^{\infty}\right) \cap F_{2}\left(\Gamma^{\infty}\right)$ is a singleton. The open set $\widetilde{\Omega}$ is not a $\epsilon-\delta$ domain. Indeed, take the point $X=(0, \beta / 2)$ and call $A_{n}=\left(F_{1} \circ F_{2}^{n}\right)(X), B_{n}=\left(F_{2} \circ F_{1}^{n}\right)(X)$, we have that

- $\lim _{n \rightarrow \infty} A_{n}=\lim _{n \rightarrow \infty} B_{n}=(0,2 \beta)$, therefore $\lim _{n \rightarrow \infty} \operatorname{dist}\left(A_{n}, B_{n}\right)=0$,

- $A_{n} \in \widetilde{\Omega}$ and $B_{n} \in \widetilde{\Omega}$,

- the length of any curve joining $A_{n}$ and $B_{n}$ that is contained in $\widetilde{\Omega}$, is greater than $2 \beta$.

As explained in $\S 2.1 .3$, one can construct a different domain $\Omega$ whose boundary contains $\Gamma^{\infty}$ by choosing for example $Y^{0}$ as a T-shaped domain, see Figure 2 .

\subsubsection{Example 2}

We make the choice $\theta=\pi / 4, \alpha=1-a / \sqrt{2}, \beta=1+a / \sqrt{2}$, so the similitudes $F_{i}, i=1,2$ read

$$
F_{i}(x)=\left(\begin{array}{l}
(-1)^{i}\left(1-\frac{a}{\sqrt{2}}\right)+\frac{a}{\sqrt{2}}\left(x_{1}+(-1)^{i} x_{2}\right) \\
1+\frac{a}{\sqrt{2}}+\frac{a}{\sqrt{2}}\left(x_{2}+(-1)^{i+1} x_{1}\right)
\end{array}\right) .
$$




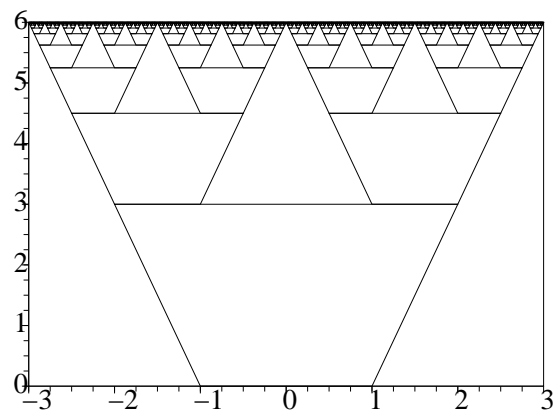

Figure 1: The ramified domain $\widetilde{\Omega}$ for the critical value $a=a^{*}=1 / 2$, and for $\alpha=3 / 2, \beta=3$.

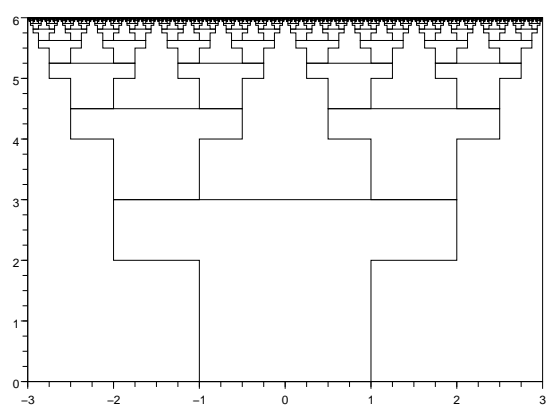

Figure 2: An example of a possible ramified domain $\Omega$ for the critical value $a=a^{*}=1 / 2$.
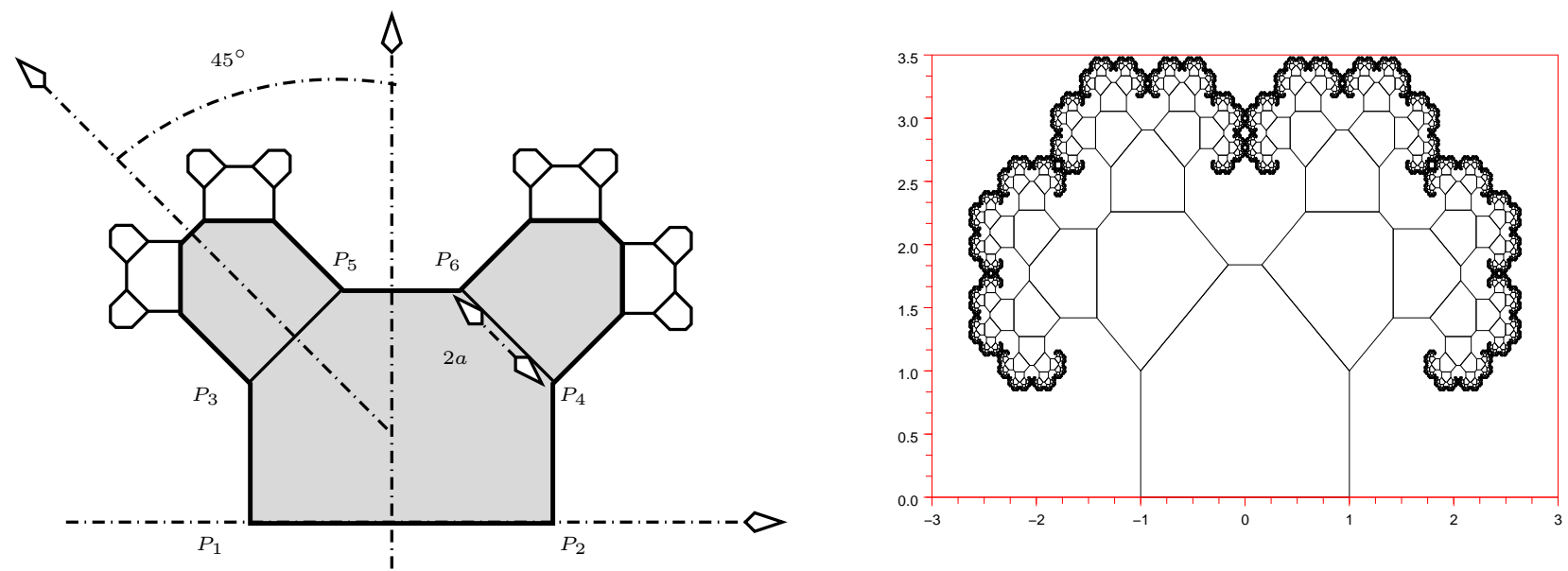

Figure 3: $\theta=\pi / 4, a=a^{*}, \alpha=1-a^{*} / \sqrt{2}, \beta=1+a^{*} / \sqrt{2}$. Left, the construction (more exactly $\left.\widetilde{Y}^{3}\right)$. Right, the ramified domain $\widetilde{\Omega}$. 
The critical parameter $a^{*}$ is the unique positive solution of $X^{3}+\sqrt{2} X^{2}-\sqrt{2} / 2=0$, i.e. $a \leq$ $a^{*} \simeq 0.593465$. The construction described in $\S 2.1 .2$ with the critical value $a=a^{*}$ leads to the domain $\widetilde{\Omega}$ shown in Figure 3 .

If $a>1 / 2$, the Hausdorff dimension of $\Gamma^{\infty}$ is larger than one. For instance, if $a=a^{*}$, then $\operatorname{dim}_{H}\left(\Gamma^{\infty}\right) \simeq 1.3284371$.

In the case when $a<a^{*}$, it can be proved that $F_{1}\left(\Gamma^{\infty}\right) \cap F_{2}\left(\Gamma^{\infty}\right)$ is empty and that $\Omega$ is a $\epsilon-\delta$ domain, see [2].

In the critical case when $a=a^{*}$, it can be proved, see [2], that $F_{1}\left(\Gamma^{\infty}\right) \cap F_{2}\left(\Gamma^{\infty}\right) \subset \Gamma^{\infty}$ is a non countable set, whose Hausdorff dimension is half the Hausdorff dimension of $\Gamma^{\infty}$. The set $\Gamma^{\infty}$ is not post-critically finite. It was also proved in [2] that $\widetilde{\Omega}$ is not a $\epsilon-\delta$ domain.

\subsection{The self-similar measure $\mu$}

For defining traces on $\Gamma^{\infty}$, we recall the classical result on self-similar measures, see $[5,7]$ and [13] page 26:

Theorem 4 There exists a unique Borel regular probability measure $\mu$ on $\Gamma^{\infty}$ such that for any Borel set $A \subset \Gamma^{\infty}$,

$$
\mu(A)=\frac{1}{2} \mu\left(F_{1}^{-1}(A)\right)+\frac{1}{2} \mu\left(F_{2}^{-1}(A)\right) .
$$

The measure $\mu$ is called the self-similar measure defined in the self-similar triplet $\left(\Gamma^{\infty}, F_{1}, F_{2}\right)$.

Proposition 2 The measure $\mu$ is a $d$-measure on $\Gamma^{\infty}$, with $d=-\log 2 / \log a$, according to the definition in [11], page 28: there exists two positive constants $c_{1}$ and $c_{2}$ such that

$$
c_{1} r^{d} \leq \mu(B(x, r)) \leq c_{2} r^{d}
$$

for any $r 0<r<1$ and $x \in \Gamma^{\infty}$, where $B(x, r)$ is the Euclidean ball in $\Gamma^{\infty}$ centered at $x$ and with radius $r$. In other words the closed set $\Gamma^{\infty}$ is a d-set, see [11], page 28.

Proof. The proof stems from the Moran condition in $\S 2.1 .2$. It is due to Moran [18] and has been extended by Kigami, see [13], §1.5, especially Proposition 1.5.8 and Theorem 1.5.7.

Remark 5 In the first example, $\S$ 2.2.1, with $a=1 / 2$, the measure $\mu$ is such that $6 \mu$ is the one-dimensional Lebesgue measure on the straight line $\Gamma^{\infty}$.

We define $L_{\mu}^{p}, p \in[1,+\infty)$ as the space of the measurable functions $v$ on $\Gamma^{\infty}$ such that $\int_{\Gamma^{\infty}}|v|^{p} d \mu<\infty$, endowed with the norm $\|v\|_{L_{\mu}^{p}}=\left(\int_{\Gamma^{\infty}}|v|^{p} d \mu\right)^{1 / p}$. We also introduce $L_{\mu}^{\infty}$, the space of essentially bounded functions with respect to the measure $\mu$. A Hilbertian basis of $L_{\mu}^{2}$ can be constructed with e.g. Haar wavelets.

\section{The space $W^{1, q}(\Omega)$}

Hereafter, the parameters $(\alpha, \beta)$ satisfy Assumption 1. Except when explicitly mentioned, we will not distinguish between the simplest geometries presented in $\S 2.1 .2$ and the more general ones introduced in $\S$ 2.1.3. The former can be seen as a special case of the latter. Therefore, we will use the notation $\Omega$ for all the domains constructed in $\S 2$.

For a real number $q \geq 1$, let $W^{1, q}(\Omega)$ be the space of functions in $L^{q}(\Omega)$ with first order partial derivatives in $L^{q}(\Omega)$. We also define $\mathcal{V}_{q}(\Omega)=\left\{v \in W^{1, q}(\Omega) ;\left.v\right|_{\Gamma^{0}}=0\right\}$ and $\mathcal{V}_{q}\left(Y^{n}\right)=$ $\left\{v \in W^{1, q}\left(Y^{n}\right) ;\left.v\right|_{\Gamma^{0}}=0\right\}$. 
If $a<1 / 2$ then $d<1$. In this case, $\Gamma^{\infty}$ is totally disconnected, see [6], Lemma 4.1 page 54 . This implies that $F_{1}\left(\Gamma^{\infty}\right) \cap F_{2}\left(\Gamma^{\infty}\right)=\emptyset$, see [13], theorem 1.6.2 page 33. Therefore, $a<a^{*}$ and we can apply the result stated in the introduction: if $q>\max (1,2-d)$, then the space of the traces on $\Gamma^{\infty}$ of the functions $v \in W^{1, q}(\Omega)$ (see $\S 3$ below for the definition) is $B_{1-\frac{2-d}{q}}^{q, q}\left(\Gamma^{\infty}\right.$ ) (see the introduction for the definition) and we will see in Theorem 8 below that in this case, $B_{1-\frac{2-d}{q}}^{q, q}\left(\Gamma^{\infty}\right)=J \operatorname{Lip}\left(1-\frac{2-d}{q}, q, q ; 0 ; \Gamma^{\infty}\right)$.

The case $a<1 / 2$ being understood, hereafter, we focus on $a$ such that $1 / 2 \leq a \leq a^{*}$, so the Hausdorff dimension $d$ of $\Gamma^{\infty}$ is not smaller than 1.

The results stated below are important for the study of elliptic boundary value problems in $\Omega$. Section 3.1 contains some Poincaré inequalities and $\S 3.2$ deals with the construction of a trace operator on $\Gamma^{\infty}$. We refer to [1] for the proofs.

We will sometimes use the notation $\lesssim$ to indicate that there may arise constants in the estimates, which are independent of the index $n$ in $\Omega^{n}$ (recall that $\Omega^{n}$ is the union of all $\mathcal{M}_{\sigma}(\Omega), \sigma \in \mathcal{A}_{n}$ ) or $\Gamma^{n}$ ( $\Gamma^{n}$ is the union of all $\left.\mathcal{M}_{\sigma}\left(\Gamma^{0}\right), \sigma \in \mathcal{A}_{n}\right), Y^{n}=Y^{0} \cup \bigcup_{1 \leq p \leq n} \bigcup_{\sigma \in \mathcal{A}_{p}} \mathcal{M}_{\sigma}\left(Y^{0}\right)$, or the index $\sigma$ in $\Omega^{\sigma}=\mathcal{M}_{\sigma}(\Omega)$ or $\Gamma^{\sigma}=\mathcal{M}_{\sigma}\left(\Gamma^{0}\right)$.

\subsection{Poincaré inequality and consequences}

Theorem 5 There exists a constant $C>0$, such that

$$
\forall v \in \mathcal{V}_{q}(\Omega), \quad\|v\|_{L^{q}(\Omega)}^{q} \leq C\|\nabla v\|_{L^{q}(\Omega)}^{q} .
$$

Corollary 1 There exists a positive constant $C$ such that for all $v \in W^{1, q}(\Omega)$,

$$
\|v\|_{L^{q}(\Omega)}^{q} \leq C\left(\|\nabla v\|_{L^{q}(\Omega)}^{q}+\left\|\left.v\right|_{\Gamma^{0}}\right\|_{L^{q}\left(\Gamma^{0}\right)}^{q}\right) .
$$

Corollary 2 There exists a positive constant $C$ such that for all integer $n \geq 0$ and for all $\sigma \in \mathcal{A}_{n}$, for all $v \in W^{1, q}\left(\Omega^{\sigma}\right)$,

$$
\|v\|_{L^{q}\left(\Omega^{\sigma}\right)}^{q} \leq C\left(a^{q n}\|\nabla v\|_{L^{q}\left(\Omega^{\sigma}\right)}^{q}+a^{n}\left\|\left.v\right|_{\Gamma^{\sigma}}\right\|_{L^{q}\left(\Gamma^{\sigma}\right)}^{q}\right),
$$

and for all $v \in W^{1, q}\left(\Omega^{n}\right)$

$$
\|v\|_{L^{q}\left(\Omega^{n}\right)}^{q} \leq C\left(a^{q n}\|\nabla v\|_{L^{q}\left(\Omega^{n}\right)}^{q}+a^{n}\left\|\left.v\right|_{\Gamma^{n}}\right\|_{L^{q}\left(\Gamma^{n}\right)}^{q}\right) .
$$

We need to estimate $\|v\|_{L^{q}\left(\Omega^{n}\right)}^{q}$ when $v \in W^{1, q}(\Omega)$ :

Lemma 1 There exists a positive constant $C$ such that for all $v \in W^{1, q}(\Omega)$, for all $n \geq 0$,

$$
\|v\|_{L^{q}\left(\Omega^{n}\right)}^{q} \leq C\left(2 a^{2}\right)^{n}\left(\|\nabla v\|_{L^{q}(\Omega)}^{q}+\left\|\left.v\right|_{\Gamma^{0}}\right\|_{L^{q}\left(\Gamma^{0}\right)}^{q}\right) .
$$

Since $2 a^{2}<1,(14)$ implies the Rellich type theorem:

Theorem 6 (Compactness) The imbedding of $W^{1, q}(\Omega)$ in $L^{q}(\Omega)$ is compact.

The following lemma will be useful for defining a trace operator on $\Gamma^{\infty}$ :

Lemma 2 There exists a positive constant $C$ such that $\forall v \in W^{1, q}(\Omega)$, for all integers $p \geq 0$,

$$
\sum_{\sigma \in \mathcal{A}_{p}} \int_{\Gamma^{\sigma}}\left(\left.v\right|_{\Gamma^{\sigma}}\right)^{q} \leq C(2 a)^{p}\left(\|\nabla v\|_{L^{q}(\Omega)}^{q}+\|v\|_{L^{q}(\Omega)}^{q}\right) .
$$


Remark 6 Note that $\left|\Gamma^{p}\right|=(2 a)^{p}\left|\Gamma^{0}\right|$, so (15) is equivalent to

$$
\frac{1}{\left|\Gamma^{p}\right|} \sum_{\sigma \in \mathcal{A}_{p}} \int_{\Gamma^{\sigma}}\left(\left.v\right|_{\Gamma^{\sigma}}\right)^{q} \lesssim\|\nabla v\|_{L^{q}(\Omega)}^{q}+\|v\|_{L^{q}(\Omega)}^{q} .
$$

Corollary 3 There exists a constant $C>0$ such that for all $v \in W^{1, q}(\Omega)$ and all integers $p \geq 0$,

$$
\sum_{\sigma \in \mathcal{A}_{p}} \int_{\Gamma^{\sigma}}\left(\left.v\right|_{\Gamma^{\sigma}}-\left\langle\left. v\right|_{\Gamma^{0}}\right\rangle\right)^{q} \leq C(2 a)^{p}\|\nabla v\|_{L^{q}(\Omega)}^{q},
$$

where $\left\langle\left. v\right|_{\Gamma^{0}}\right\rangle$ is the mean value of $\left.v\right|_{\Gamma^{0}}$ on $\Gamma^{0}$.

Remark 7 Similar results in a different geometry were proved by Berger [3] with different methods.

\subsection{A trace operator on $\Gamma^{\infty}$}

We construct a sequence $\left(\ell^{n}\right)_{n}$ of approximations of the trace operator: consider the sequence of linear operators $\ell^{n}: W^{1, q}(\Omega) \rightarrow L_{\mu}^{q}$,

$$
\ell^{n}(v)=\sum_{\sigma \in \mathcal{A}_{n}}\left(\frac{1}{\left|\Gamma^{\sigma}\right|} \int_{\Gamma^{\sigma}} v d x\right) \mathbf{1}_{\mathcal{M}_{\sigma}\left(\Gamma^{\infty}\right)},
$$

where $\left|\Gamma^{\sigma}\right|$ is the one-dimensional Lebesgue measure of $\Gamma^{\sigma}$, see [1].

Proposition 3 The sequence $\left(\ell^{n}\right)_{n}$ converges in $\mathcal{L}\left(W^{1, q}(\Omega), L_{\mu}^{q}\right)$ to an operator that we call $\ell^{\infty}$.

Proof. See [1].

\section{Lipschitz Functions with Jumps on $\Gamma^{\infty}$}

In [10], A. Jonsson has introduced Haar wavelets of arbitrary order on self-similar fractal sets and has used these wavelets for constructiong a family of Lipschitz spaces. These function spaces are named $J \operatorname{Lip}(\alpha, p, q ; m ; K)$, where $K$ is the fractal set, $\alpha$ is a nonnegative real number, $p, q$ are two real numbers not smaller than 1 and $m$ is an integer ( $m$ is the order of the Haar wavelets used for constructing the space). Here $J$ stands for jumps, since the considered functions may jump at some points of $K$. If the fractal set $K$ is totally disconnected, then these spaces coincide with the Lipschitz spaces $\operatorname{Lip}(\alpha, p, q ; m ; K)$ also introduced in [10]. The latter are a generalization of the more classical spaces $\operatorname{Lip}(\alpha, p, q ; K)$ introduced in [11] since $\operatorname{Lip}(\alpha, p, q ;[\alpha] ; K)=\operatorname{Lip}(\alpha, p, q ; K)$. Note that $\operatorname{Lip}(\alpha, p, q ;[\alpha] ; K)=B_{\alpha}^{p, q}(K)$, see[12].

Since this is sufficient for what follows, we will focus on the case when $K=\Gamma^{\infty}$ and $p=q$.

\subsection{Definition of $\operatorname{JLip}\left(\alpha, q, q ; 0 ; \Gamma^{\infty}\right)$ for $0<\alpha<1$.}

We review the theory proposed in [10] in the special case when $0<\alpha<1$ and $m=0$. Here, since we focus on the case $m=0$, we do not need to suppose that $\Gamma^{\infty}$ is not contained in a straight line, as it was done in [10] in order to obtain that the fractal set has a Markov property. For $f \in L_{\mu}^{q}$, a positive integer $n$ and $\sigma \in \mathcal{A}_{n}$, we define $P_{\sigma} f$ as the projection of $f$ on constants:

$$
P_{\sigma} f=2^{n} \int_{\mathcal{M}_{\sigma}\left(\Gamma^{\infty}\right)} f d \mu .
$$


We also define $P_{0} f$ as the projection of $f$ on constants:

$$
P_{0} f=\int_{\Gamma^{\infty}} f d \mu
$$

For an integer $\nu$, we define the set $J_{\nu}$ :

$$
J_{\nu}=\left\{p \in \mathbb{N}, \text { s.t. } \forall \sigma \in \mathcal{A}_{p}, 2^{-\nu} \leq \operatorname{diam}\left(\mathcal{M}_{\sigma}\left(\Gamma^{\infty}\right)\right)<2^{-\nu+1}\right\},
$$

agreeing that $\mathcal{M}_{\sigma}=I d$ if $p=0, \sigma \in \mathcal{A}_{p}$.

Let $\nu_{0}$ be the integer (possibly negative) such that $0 \in J_{\nu_{0}}$.

Consider a real number $\alpha, 0<\alpha<1$. For $f \in L_{\mu}^{2}$ and an integer $\nu \geq \nu_{0}$, we define $A_{\nu}(f)$ :

$$
\begin{aligned}
& A_{\nu_{0}}(f)=2^{\alpha \nu_{0}}\left(\int_{\Gamma^{\infty}}\left|f-P_{0} f\right|^{q} d \mu+\sum_{p \in J_{\nu_{0}}, p>0} \sum_{\sigma \in \mathcal{A}_{p}} \int_{\mathcal{M}_{\sigma}\left(\Gamma^{\infty}\right)}\left|f-P_{\sigma} f\right|^{q} d \mu\right)^{1 / q}, \\
& A_{\nu}(f)=2^{\alpha \nu}\left(\sum_{p \in J_{\nu}} \sum_{\sigma \in \mathcal{A}_{p}} \int_{\mathcal{M}_{\sigma}\left(\Gamma^{\infty}\right)}\left|f-P_{\sigma} f\right|^{q} d \mu\right)^{1 / q}, \quad \text { if } \nu>\nu_{0} .
\end{aligned}
$$

Definition 1 The function $f$ belongs to $\operatorname{JLip}\left(\alpha, q, q ; 0 ; \Gamma^{\infty}\right)$ if the norm

$$
\|f\|_{J \operatorname{Lip}\left(\alpha, q, q ; 0 ; \Gamma^{\infty}\right)}=\left(\|f\|_{L_{\mu}^{q}}^{q}+\sum_{\nu=\nu_{0}}^{\infty} A_{\nu}^{q}(f)\right)^{\frac{1}{q}}
$$

is finite.

Following [10], it is possible to characterize $\operatorname{Lip}\left(\alpha, q, q ; 0 ; \Gamma^{\infty}\right)$ by using expansions in the standard Haar wavelet basis on $\Gamma^{\infty}$. Consider the Haar mother wavelet $g^{0}$ on $\Gamma^{\infty}$,

$$
g^{0}=1_{F_{1}\left(\Gamma^{\infty}\right)}-1_{F_{2}\left(\Gamma^{\infty}\right)},
$$

and for $n \in \mathbb{N}, n>0, \sigma \in \mathcal{A}_{n}$, let $g^{\sigma}$ be given by

$$
\left.g^{\sigma}\right|_{\mathcal{M}_{\sigma}\left(\Gamma^{\infty}\right)}=2^{n / 2} g^{0} \circ \mathcal{M}_{\sigma}^{-1}, \quad \text { and }\left.\quad g^{\sigma}\right|_{\Gamma^{\infty} \backslash \mathcal{M}_{\sigma}\left(\Gamma^{\infty}\right)}=0 .
$$

It is proved in [9] $\S 5$ that a function $f \in L_{\mu}^{q}$ can be expanded on the Haar basis as follows:

$$
f=P_{0} f+\beta_{0} g^{0}+\sum_{n \geq 1} \sum_{\sigma \in \mathcal{A}_{n}} \beta_{n, \sigma} g^{\sigma} .
$$

Let $b_{0}$ be a real number and $\left(b_{n, \sigma}\right)_{n \geq 1, \sigma \in \mathcal{A}_{n}}$ be a sequence of real numbers, we define $\left\|\left(b_{0}, b_{n, \sigma}\right)\right\|_{b_{\alpha}^{q, q}}$ :

$$
\left\|\left(b_{0}, b_{n, \sigma}\right)\right\|_{b_{\alpha}^{q, q}}=\left(\begin{array}{l}
2^{\alpha \nu_{0} q} 2^{d \nu_{0}\left(\frac{1}{2}-\frac{1}{q}\right) q}\left(\left|b_{0}\right|^{q}+\sum_{p \in J_{\nu_{0}, p>0}} \sum_{\sigma \in \mathcal{A}_{p}}\left|b_{p, \sigma}\right|^{q}\right) \\
+\sum_{\nu=\nu_{0}+1}^{\infty} 2^{\alpha \nu q} 2^{d \nu\left(\frac{1}{2}-\frac{1}{q}\right) q} \sum_{p \in J_{\nu}} \sum_{\sigma \in \mathcal{A}_{p}}\left|b_{p, \sigma}\right|^{q}
\end{array}\right)^{\frac{1}{q}}
$$

Theorem 7 (Jonsson) A function $f \in L_{\mu}^{q}$ belongs to $\operatorname{JLip}\left(\alpha, q, q ; 0 ; \Gamma^{\infty}\right)$ if and only if the coefficients of its expansion in the Haar wavelets basis satisfy $\left|P_{0} f\right|+\left\|\left(\beta_{0}, \beta_{n, \sigma}\right)\right\|_{b_{\alpha}^{q, q}}$ is finite; this defines a norm in $\operatorname{Jip}\left(\alpha, q, q ; 0 ; \Gamma^{\infty}\right)$ equivalent to the one in (19). 
Proof. This is a particular case of Theorem 1 in [10].

If the fractal set $\Gamma^{\infty}$ is totally disconnected, then the jumps which are allowed in the space $J \operatorname{Lip}\left(\alpha, q, q ; 0 ; \Gamma^{\infty}\right)$ disappear:

Theorem 8 (Jonsson) If $F_{1}\left(\Gamma^{\infty}\right) \cap F_{2}\left(\Gamma^{\infty}\right)$ is empty, then

$$
J \operatorname{Lip}\left(\alpha, q, q ; 0 ; \Gamma^{\infty}\right)=\operatorname{Lip}\left(\alpha, q, q ; 0 ; \Gamma^{\infty}\right)=B_{\alpha}^{q, q}\left(\Gamma^{\infty}\right),
$$

where the Lipschitz space Lip $\left(\alpha, q, q ; 0 ; \Gamma^{\infty}\right)$ and the Besov space $B_{\alpha}^{q, q}\left(\Gamma^{\infty}\right)$ are defined in [11].

Proof. This is a particular case of Theorem 2 in [10], see also [9] for a partial proof.

Let us assume that $\operatorname{diam}\left(\Gamma^{\infty}\right)>0$. Since in our case the ratii of the similitudes $F_{1}$ and $F_{2}$ both take the value $a$, we see that $\operatorname{diam}\left(\mathcal{M}_{\sigma}\left(\Gamma^{\infty}\right)\right)=a^{n} \operatorname{diam}\left(\Gamma^{\infty}\right)$ if $\sigma \in \mathcal{A}_{n}$ and $n>0$. Therefore, for $n>0, n \in J_{\nu}$ if and only if

$$
2^{-\nu} \leq a^{n} \operatorname{diam}\left(\Gamma^{\infty}\right)<2^{-\nu+1}
$$

which can be written

$$
-\nu \leq n \frac{\log a}{\log 2}+\frac{\log \left(\operatorname{diam}\left(\Gamma^{\infty}\right)\right)}{\log 2}<-\nu+1
$$

or

$$
-\nu=\left[n \frac{\log a}{\log 2}+\frac{\log \left(\operatorname{diam}\left(\Gamma^{\infty}\right)\right)}{\log 2}\right]=\left[-n / d+\frac{\log \left(\operatorname{diam}\left(\Gamma^{\infty}\right)\right)}{\log 2}\right]
$$

where $d=-\log 2 / \log a$ is the Hausdorff dimension of $\Gamma^{\infty}$ and [.] stands for the integer part. Let $b_{0}$ be a real number and $\left(b_{n, \sigma}\right)_{n \geq 1, \sigma \in \mathcal{A}_{n}}$ be a sequence of real numbers; we define $\left\|\left(b_{0}, b_{n, \sigma}\right)\right\|_{\tilde{b}_{\alpha}^{q, q}}$ :

$$
\begin{aligned}
\left\|\left(b_{0}, b_{n, \sigma}\right)\right\|_{\tilde{b}_{\alpha}^{q, q}} & =\left(\left|b_{0}\right|^{q}+\sum_{n=1}^{\infty} 2^{q \alpha n / d} 2^{n(1 / 2-1 / q) q} \sum_{\sigma \in \mathcal{A}_{n}}\left|b_{n, \sigma}\right|^{q}\right)^{\frac{1}{q}} . \\
& =\left(\left|b_{0}\right|^{q}+\sum_{n=1}^{\infty} a^{-q \alpha n} 2^{n(1 / 2-1 / q) q} \sum_{\sigma \in \mathcal{A}_{n}}\left|b_{n, \sigma}\right|^{q}\right)^{\frac{1}{q}} .
\end{aligned}
$$

Assuming that $\operatorname{diam}\left(\Gamma^{\infty}\right) \neq 0$, there exist two positive constants $c_{1}$ and $c_{2}$ such that

$$
c_{1}\left\|\left(b_{0}, b_{n, \sigma}\right)\right\|_{b_{\alpha}^{q, q}} \leq\left\|\left(b_{0}, b_{n, \sigma}\right)\right\|_{\tilde{b}_{\alpha}^{q, q}} \leq c_{2}\left\|\left(b_{0}, b_{n, \sigma}\right)\right\|_{b_{\alpha}^{q, q}}
$$

for any sequence $\left(b_{n, \sigma}\right)_{n \geq 1, \sigma \in \mathcal{A}_{n}}$ such that $\left|b_{0}\right|^{q}+\sum_{n=1}^{\infty} 2^{n(1 / 2-1 / q) q} \sum_{\sigma \in \mathcal{A}_{n}}\left|b_{n, \sigma}\right|^{q}<\infty$. We have the corollary of Theorem 7:

Corollary 4 A function $f \in L_{\mu}^{q}$ belongs to $\operatorname{JLip}\left(\alpha, q, q ; 0 ; \Gamma^{\infty}\right)$ if and only if the coefficients of its expansion in the Haar wavelets basis satisfy $\left|P_{0} f\right|+\left\|\left(\beta_{0}, \beta_{n, \sigma}\right)\right\|_{\tilde{b}_{\alpha}^{q, q}}$ is finite; the norm of $f$ in JLip $\left(\alpha, q, q ; 0 ; \Gamma^{\infty}\right)$ is equivalent to this sum. Another equivalent norm is

$$
\left(\|f\|_{L_{\mu}^{q}}^{q}+\int_{\Gamma^{\infty}}\left|f-P_{0} f\right|^{q} d \mu+\sum_{n=1}^{\infty} a^{-\alpha q n} \sum_{\sigma \in \mathcal{A}_{n}} \int_{\mathcal{M}_{\sigma}\left(\Gamma^{\infty}\right)}\left|f-P_{\sigma} f\right|^{q} d \mu\right)^{\frac{1}{q}}
$$


Remark 8 In the special case when $\alpha=1-(2-d) / q$,

$$
\left\|\left(b_{0}, b_{n, \sigma}\right)\right\|_{\tilde{b}_{\alpha}^{q, q}}=\left(\left|b_{0}\right|^{q}+\sum_{n=1}^{\infty} 2^{\frac{n q}{2}} a^{-n(q-2)} \sum_{\sigma \in \mathcal{A}_{n}}\left|b_{n, \sigma}\right|^{q}\right)^{\frac{1}{q}}
$$

and the norm in (26) reads

$$
\left(\|f\|_{L_{\mu}^{q}}^{q}+\int_{\Gamma^{\infty}}\left|f-P_{0} f\right|^{q} d \mu+\sum_{n=1}^{\infty}\left(2 a^{2-q}\right)^{n} \sum_{\sigma \in \mathcal{A}_{n}} \int_{\mathcal{M}_{\sigma}\left(\Gamma^{\infty}\right)}\left|f-P_{\sigma} f\right|^{q} d \mu\right)^{\frac{1}{q}} .
$$

These observations will be useful in the proof of Theorem 9 below.

\subsection{Definition of $J \operatorname{Lip}\left(\alpha, q, q ; m ; \Gamma^{\infty}\right)$ for a positive integer $m$ and $m \leq \alpha<m+1$.}

Following [10], we suppose that $\Gamma^{\infty}$ is not contained in a straight line (this rules out the geometry presented in $\S 2.2 .1$ above). This assumption implies that $\Gamma^{\infty}$ preserves Markov's inequality, see [10].

Call $\mathcal{S}_{0}$ the subspace of $L_{\mu}^{2}$ made of polynomials of order not larger than $m$; thanks to Markov's inequality, its dimension is $M_{0}=(m+2)(m+1) / 2$; let $\left(\phi^{i}\right)_{1 \leq i \leq M_{0}}$ be a basis of $\mathcal{S}_{0}$ orthonormal with respect to $(\cdot, \cdot)_{L_{\mu}^{2}}$. For $f \in L_{\mu}^{1}$, we define by $P_{0}^{(m)} f$ the projection of $f$ onto $\mathcal{P}_{m}$ defined as follows: let $\left(\phi^{j}\right)_{1 \leq j \leq M_{0}}$ be an orthonormal basis of $\mathcal{S}_{0}$ and $P_{0}^{(m)} f=\sum_{i} \phi^{i} \int_{\Gamma^{\infty}} f \phi^{i} d \mu$.

Similarly, for $f \in L_{\mu}^{1}\left(\mathcal{M}_{\sigma}\left(\Gamma^{\infty}\right)\right)$, we define by $P_{\sigma}^{(m)} f$ the projection of $f$ onto $\mathcal{P}_{m}$ :

$$
P_{\sigma}^{(m)} f=\left(P_{0}^{(m)}\left(f \circ \mathcal{M}_{\sigma}\right)\right) \circ \mathcal{M}_{\sigma}^{-1} .
$$

The following definition of the space $\operatorname{JLip}\left(\alpha, q, q ; m ; \Gamma^{\infty}\right)$ has to be compared to (26):

Definition 2 Let $m$ be a positive integer and $\alpha$ be a real number such that $m \leq \alpha<m+1$. The function $f \in L_{\mu}^{q}$ belongs to $\operatorname{JLip}\left(\alpha, q, q ; m ; \Gamma^{\infty}\right)$ if the norm

$\|f\|_{J L i p\left(\alpha, q, q ; m ; \Gamma^{\infty}\right)}=\left(\|f\|_{L_{\mu}^{q}}^{q}+\int_{\Gamma^{\infty}}\left|f-P_{0}^{(m)} f\right|^{q} d \mu+\sum_{n=1}^{\infty} a^{-\alpha q n} \sum_{\sigma \in \mathcal{A}_{n}} \int_{\mathcal{M}_{\sigma}\left(\Gamma^{\infty}\right)}\left|f-P_{\sigma}^{(m)} f\right|^{q} d \mu\right)^{\frac{1}{q}}$.

is finite.

Haar wavelets of order $m$ can be used for an equivalent definition of $\operatorname{Lip}\left(\alpha, q, q ; m ; \Gamma^{\infty}\right)$ : call $\mathcal{S}_{1}$ the space of the functions $f$ defined on $\Gamma^{\infty}$ such that $f$ coincides with a polynomial of degree $m$ on $F_{i}\left(\Gamma^{\infty}\right) \backslash\left(F_{1}\left(\Gamma^{\infty}\right) \cap F_{2}\left(\Gamma^{\infty}\right)\right), i=1,2$. The dimension of $\mathcal{S}_{1}$ is $2 M_{0}$. The orthogonal complement $\mathcal{S}_{1} \ominus \mathcal{S}_{0}$ of $\mathcal{S}_{0}$ in $\mathcal{S}_{1}$ has dimension $M_{0}$. Choose an orthonormal basis $\left(g^{0, i}\right)_{1 \leq i \leq M_{0}}$ of $\mathcal{S}_{1} \ominus \mathcal{S}_{0}$. Finally, for $n \geq 1$ and $\sigma \in \mathcal{A}_{n}$, define

$$
g^{\sigma, i}=2^{n / 2} g^{0, i} \circ \mathcal{M}_{\sigma}^{-1} .
$$

The family $\left(\phi^{i}, g^{0, i}, g^{\sigma, i}\right), 1 \leq i \leq M_{0}, \sigma \in \mathcal{A}_{n}, n \geq 1$ is an orthonormal and complete family of $L_{\mu}^{2}$. It is proved in [10] that a function $f \in L_{\mu}^{q}$ can be expanded as follows

$$
f=\sum_{i=1}^{M_{0}} \lambda_{i} \phi^{i}+\sum_{i=1}^{M_{0}} \beta_{0, i} g^{0, i}+\sum_{i=1}^{M_{0}} \sum_{n \geq 1} \sum_{\sigma \in \mathcal{A}_{n}} \beta_{n, \sigma, i} g^{\sigma, i}
$$


Proposition 4 (Jonsson, [10], Th. 1) A function $f \in L_{\mu}^{q}$ belongs to JLip $\left(\alpha, q, q ; m ; \Gamma^{\infty}\right)$ if and only if

$$
\left(\sum_{i=1}^{M_{0}}\left|\lambda_{i}\right|^{q}+\sum_{i=1}^{M_{0}}\left|\beta_{0, i}\right|^{q}+\sum_{i=1}^{M_{0}} \sum_{n=1}^{\infty} a^{-q \alpha n} 2^{n(1 / 2-1 / q) q} \sum_{\sigma \in \mathcal{A}_{n}}\left|\beta_{n, \sigma, i}\right|^{q}\right)^{\frac{1}{q}}<\infty,
$$

where $\lambda_{i}, \beta_{0, i}, \beta_{n, \sigma, i}$ are the coefficients in (28). The norm in (29) is equivalent to that in (27).

\section{Traces of functions belonging to $W^{1, q}(\Omega)$}

We consider the case when $1 / 2 \leq a \leq a^{*}$; we are going to give a trace theorem with a direct proof (which differs from the previous argument when $a<a^{*}$ ).

The main theorem is as follows:

Theorem 9 If $1 / 2 \leq a \leq a^{*}$, then for all $q, 1<q<\infty$,

$$
\ell^{\infty}\left(W^{1, q}(\Omega)\right)=J \operatorname{Lip}\left(1-\frac{2-d}{q}, q, q ; 0 ; \Gamma^{\infty}\right) .
$$

Proof. Theorem 9 is a straightforward consequence of Proposition 5 and Theorem 10 below.

Remark 9 Note the contrast with the fact that functions in $W^{1, q}\left(\mathbb{R}^{2}\right)$ have their traces in $\operatorname{Lip}\left(1-(2-d) / q, q, q ; 0 ; \Gamma^{\infty}\right)$, see [11] page 182 and [9, 10].

Proposition 5 For all real number $q \geq 1$, there exists a constant $C$ such that for all $v \in$ $J \operatorname{Lip}\left(1-\frac{2-d}{q}, q, q ; 0 ; \Gamma^{\infty}\right)$, there exists $\tilde{v} \in \mathcal{V}_{q}(\Omega)$ with the following properties: $\ell^{\infty}(\tilde{v})=v$, and

$$
\|\tilde{v}\|_{\mathcal{V}_{q}(\Omega)}^{q} \approx \int_{\Omega}|\nabla \tilde{v}|^{q} \leq C\|v\|_{J \operatorname{Lip}\left(1-\frac{2-d}{q}, q, q ; 0 ; \Gamma^{\infty}\right)}^{q} .
$$

Proof. First, let us consider the case when $v$ is a Haar wavelet on $\Gamma^{\infty}$. We start with the mother wavelet $g^{0}$. One can construct a function $\tilde{g}^{0}$ such that $\tilde{g}^{0} \in \mathcal{V}_{q}(\Omega)$ for all $1 \leq q<\infty$ and

$$
\left.\tilde{g}^{0}\right|_{F_{1}(\Omega)}=-1 \quad \text { and } \quad \tilde{g}^{0}\left(-x_{1}, x_{2}\right)=-\tilde{g}^{0}\left(x_{1}, x_{2}\right), \quad \forall x=\left(x_{1}, x_{2}\right) \in \Omega .
$$

It is an easy matter to check that $\ell^{\infty}\left(\tilde{g}^{0}\right)=g^{0}$ and that $\int_{\Omega}\left|\nabla \tilde{g}^{0}\right|^{q}=\int_{Y^{0}}\left|\nabla \tilde{g}^{0}\right|^{q}$.

For a positive integer $n$ and $\sigma \in \mathcal{A}_{n}$, let us define the function $\tilde{g}^{\sigma}, \tilde{g}^{\sigma} \in \mathcal{V}_{q}(\Omega)$ for all $1 \leq q<\infty$, by:

$$
\begin{aligned}
\left.\tilde{g}^{\sigma}\right|_{\Omega^{\sigma}} & =2^{n / 2} \tilde{g}^{0} \circ \mathcal{M}_{\sigma}^{-1}, \\
\left.\tilde{g}^{\sigma}\right|_{\Omega \backslash \Omega^{\sigma}} & =0 .
\end{aligned}
$$

From the definition of $g^{\sigma}$, we see that $\ell^{\infty}\left(\tilde{g}^{\sigma}\right)=g^{\sigma}$. The change of variable $x=\mathcal{M}_{\sigma} y$ yields

$$
\int_{\Omega}\left|\nabla \tilde{g}^{\sigma}\right|^{q}=\int_{\Omega^{\sigma}}\left|\nabla \tilde{g}^{\sigma}\right|^{q}=2^{n \frac{q}{2}} a^{(2-q) n} \int_{\Omega}\left|\nabla \tilde{g}^{0}\right|^{q}=2^{n \frac{q}{2}} a^{(2-q) n} \int_{Y^{0}}\left|\nabla \tilde{g}^{0}\right|^{q} .
$$

Moreover, the support of $\nabla \tilde{g}^{\sigma}$ is contained in $Y^{\sigma}$, which implies that for all positive integer $n$ and $n^{\prime}$, for all $\sigma \in \mathcal{A}_{n}, \eta \in \mathcal{A}_{n^{\prime}}$,

- the supports of $\nabla \tilde{g}^{\sigma}$ and $\nabla \tilde{g}^{0}$ are disjoint.

- if $(n, \sigma) \neq\left(n^{\prime}, \eta\right)$ then the supports of $\nabla \tilde{g}^{\sigma}$ and $\nabla \tilde{g}^{\eta}$ are disjoint. 
We also introduce a function $\chi, \chi \in \mathcal{V}_{q}(\Omega)$ for all $1 \leq q<\infty$, such that $\left.\chi\right|_{\Omega^{1}}=1$ and that $\chi\left(x_{1}, x_{2}\right)=\chi\left(-x_{1}, x_{2}\right)$. It is clear that $\ell^{\infty}(\chi)=1$. Moreover, it can be seen that

$$
\left\{\begin{array}{c}
\int_{\Omega} \nabla \chi \cdot \nabla \tilde{g}^{0}=0 \quad, \\
\text { and } \quad \int_{\Omega} \nabla \chi \cdot \nabla \tilde{g}^{\sigma}=0
\end{array}\right.
$$

Consider now $v \in J \operatorname{Lip}\left(1-\frac{2-d}{q}, q, q ; 0 ; \Gamma^{\infty}\right)$. We can expand $v$ on the Haar basis:

$$
v=P_{0} v+\beta_{0} g^{0}+\sum_{n \geq 1} \sum_{\sigma \in \mathcal{A}_{n}} \beta_{n, \sigma} g^{\sigma},
$$

where $\beta_{0}=\int_{\Gamma^{\infty}} v g^{0} d \mu$ and $\beta_{n, \sigma}=\int_{\Gamma^{\infty}} v g^{\sigma} d \mu$. We know from Theorem 1 and Corollary 4 (see Remark 8) that

$$
\left|\beta_{0}\right|^{q}+\sum_{n=1}^{\infty} 2^{n \frac{q}{2}} a^{(2-q) n} \sum_{\sigma \in \mathcal{A}_{n}}\left|\beta_{n, \sigma}\right|^{q}<\infty
$$

Let us define the function $\tilde{v}: \Omega \rightarrow \mathbb{R}$, by

$$
\tilde{v}=\left(P_{0} v\right) \chi+\beta_{0} \tilde{g}^{0}+\sum_{n \geq 1} \sum_{\sigma \in \mathcal{A}_{n}} \beta_{n, \sigma} \tilde{g}^{\sigma},
$$

where we agree to identify the function $P_{0} v$ with its real value. From a classical inequality, we obtain that

$$
\int_{\Omega}|\nabla \tilde{v}|^{q} \lesssim\left|P_{0} v\right|^{q} \int_{\Omega}|\nabla \chi|^{q}+\int_{\Omega}\left|\beta_{0} \nabla \tilde{g}^{0}+\sum_{n \geq 1} \sum_{\sigma \in \mathcal{A}_{n}} \beta_{n, \sigma} \nabla \tilde{g}^{\sigma}\right|^{q} .
$$

From (35) and the observation on the supports of the functions $\nabla \tilde{g}^{\sigma}$, this yields that

$$
\int_{\Omega}|\nabla \tilde{v}|^{q} \lesssim\left|P_{0} v\right|^{q} \int_{\Omega}|\nabla \chi|^{q}+\left(\left|\beta_{0}\right|^{q}+\sum_{n \geq 1} \sum_{\sigma \in \mathcal{A}_{n}} 2^{n \frac{q}{2}} a^{(2-q) n}\left|\beta_{n, \sigma}\right|^{q}\right) \int_{Y^{0}}\left|\nabla \tilde{g}^{0}\right|^{q},
$$

which is finite from (37), and the right hand sides of the two above estimates coincide. Therefore, $\tilde{v} \in \mathcal{V}_{q}(\Omega)$, and it is an easy matter to see that $\ell^{\infty}(\tilde{v})=v$ using the continuity of $\ell^{\infty}$ in $W^{1, q}(\Omega)$, see Proposition 3. Finally, (31) is a consequence of Theorem 1 and Corollary 4, (see Remark 8).

Theorem 10 Assume that $1 / 2 \leq a \leq a^{*}$. For all $q, 1<q<\infty$ and $v \in W^{1, q}(\Omega)$, $\ell^{\infty}(v)$ belongs to JLip $\left(1-\frac{2-d}{q}, q, q ; 0 ; \Gamma^{\infty}\right)$ and there exists a constant $c$ such that

$$
\left\|\ell^{\infty}(v)\right\|_{J L i p\left(1-\frac{2-d}{q}, q, q ; 0 ; \Gamma^{\infty}\right)} \leq c\|v\|_{W^{1, q}(\Omega)}, \quad \forall v \in W^{1, q}(\Omega) .
$$

The proof of Theorem 10 is postponed to the end of $\S 5$. It makes use of the strengthened trace inequality stated in Theorem 11 below. The following lemma (see, e.g., [4], p. 126) will play an important role in the proof of Theorem 11.

Lemma 3 (Peetre-Tartar) Let $V, V_{1}, V_{2}, W$ be Banach spaces, and let $A_{i} \in \mathcal{L}\left(V, V_{i}\right)$ be continuous linear maps for $i=1,2$, the map $A_{1}$ being compact. Suppose there exists $c_{0}>0$ such that

$$
\|v\|_{V} \leq c_{0}\left(\left\|A_{1} v\right\|_{V_{1}}+\left\|A_{2} v\right\|_{V_{2}}\right), \quad \text { for any } v \in V .
$$

In addition, let $L \in \mathcal{L}(V, W)$ be a continuous linear map such that $\left.L\right|_{\text {ker } A_{2}} \equiv 0$. Then there exists $C>0$ such that

$$
\|L v\|_{W} \leq C\left\|A_{2} v\right\|_{V_{2}}, \quad \text { for any } v \in V .
$$


Theorem 11 Assume that $a \geq 1 / 2$.

1. For all real number $\rho$ such that

$$
\left(2 a^{2}\right)^{q-1}<\rho<1
$$

there exists a constant $C$ such that for all $v \in \mathcal{V}_{q}(\Omega)$,

$$
\left\|\ell^{\infty}(v)\right\|_{L_{\mu}^{q}}^{q} \leq C\left(\|\nabla v\|_{L^{q}\left(Y^{0}\right)}^{q}+\sum_{n=1}^{\infty} \rho^{-n}\left(2 a^{2}\right)^{n(q-1)} \sum_{\sigma \in \mathcal{A}_{n}}\|\nabla v\|_{L^{q}\left(\mathcal{M}_{\sigma}\left(Y^{0}\right)\right)}^{q}\right) .
$$

2. For $\rho$ satisfying (40), there exists a constant $C$ such that, for all $v \in W^{1, q}(\Omega)$,

$$
\left\|\ell^{\infty}(v)-P_{0}\left(\ell^{\infty}(v)\right)\right\|_{L_{\mu}^{q}}^{q} \leq C\left(\|\nabla v\|_{L^{q}\left(Y^{0}\right)}^{q}+\sum_{n=1}^{\infty} \rho^{-n}\left(2 a^{2}\right)^{n(q-1)} \sum_{\sigma \in \mathcal{A}_{n}}\|\nabla v\|_{L^{q}\left(\mathcal{M}_{\sigma}\left(Y^{0}\right)\right)}^{q}\right) .
$$

\section{Proof.}

The proofs in the cases when $a=1 / 2$ and $a>1 / 2$ slightly differ. Hereafter, we thoroughly discuss the case $a>1 / 2$. For the ease of the reader, the case $a=1 / 2$ will be discussed in Appendix B. In both cases, the proof of Theorem 11 is done in three steps.

The case when $a>1 / 2$

Step 1 The first step consists of proving (41) for the constructions described in $\S$ 2.1.2:

we take $P_{1}=(-1,0), P_{2}=(1,0)$, and define $P_{3}=(0, \beta+a \sin \theta)$. We partition $\widetilde{Y}^{0}$ into five nonoverlapping triangles: $\widetilde{Y}^{0}=\cup_{i=1}^{5} \widetilde{T}_{i}$, where $\widetilde{T}_{1}=\left(P_{1}, P_{2}, P_{3}\right), \widetilde{T}_{2}=\left(P_{1}, P_{3}, F_{1}\left(P_{1}\right)\right)$, $\widetilde{T}_{3}=\left(F_{1}\left(P_{1}\right), F_{1}\left(P_{2}\right), P_{3}\right), \widetilde{T}_{4}=\left(P_{2}, F_{2}\left(P_{2}\right), P_{3}\right)$ and $\widetilde{T}_{5}=\left(F_{2}\left(P_{2}\right), F_{2}\left(P_{1}\right), P_{3}\right)$, see Figure 4 .

Let us introduce the two real numbers

$$
\begin{aligned}
& r_{1}=(\alpha-1+a \cos \theta)(\beta+a \sin \theta)+(\beta-a \sin \theta) \\
& r_{2}=2 a \sin \theta(\alpha-a \cos \theta)+\beta+a \sin \theta
\end{aligned}
$$

The assumptions (5) and (6) imply that $r_{1} \geq 0$ and that $r_{2} \geq \beta+a \sin \theta$. We introduce the points $\widehat{P}_{4}=\left(-1, r_{1}\right), \widehat{P}_{5}=\left(0, r_{2}\right)$ and $\widehat{P}_{6}=\left(1, r_{1}\right)$, and the triangles $\widehat{T}_{1}=\widetilde{T}_{1}, \widehat{T}_{2}=\left(P_{1}, P_{3}, \widehat{P}_{4}\right)$, $\widehat{T}_{3}=\left(\widehat{P}_{4}, \widehat{P}_{5}, P_{3}\right), \widehat{T}_{4}=\left(P_{2}, \widehat{P}_{6}, P_{3}\right)$ and $\widehat{T}_{5}=\left(\widehat{P}_{6}, \widehat{P}_{5}, P_{3}\right)$, see Figure 4 .

The points $\widehat{P}_{i}, i=4,5,6$ have been chosen in such a way that

- The triangles $\widetilde{T}_{i}$ and $\widehat{T}_{i}$ have the same measure, $i=1, \ldots 5$.

- $\widehat{P}_{4}$ and $P_{1}$ have the same abcissa $-1, \widehat{P}_{5}$ and $P_{3}$ have the same abcissa 0 , and $\widehat{P}_{6}$ and $P_{2}$ have the same abcissa 1 .

The triangles $\widehat{T}_{i} i=1, \ldots, 5$ form a nonoverlapping partition of a new polygonal domain $\widehat{Y}_{0}$ with a vertical fracture namely $\left[P_{3}, \widehat{P}_{5}\right]$, see Figure 4 .

Therefore it is possible to construct a continuous and piecewise affine map $H$ from $\widetilde{Y}_{0}$ onto $\widehat{Y}_{0}$, in such a way that $H_{\mid \widetilde{T}_{i}}$ is an affine map from $\widetilde{T}_{i}$ onto $\widehat{T}_{i}$. It is clear that $H$ is measure preserving. Let $G_{1}$ and $G_{2}$ be the maps in $\mathbb{R}^{2}$ defined by

$$
\begin{aligned}
& G_{1}\left(x_{1}, x_{2}\right)=\left(\frac{x_{1}-1}{2}, \frac{r_{1}+r_{2}}{2}+\frac{r_{2}-r_{1}}{2} x_{1}+2 a^{2} x_{2}\right), \\
& G_{2}\left(x_{1}, x_{2}\right)=\left(\frac{x_{1}+1}{2}, \frac{r_{1}+r_{2}}{2}-\frac{r_{2}-r_{1}}{2} x_{1}+2 a^{2} x_{2}\right) .
\end{aligned}
$$



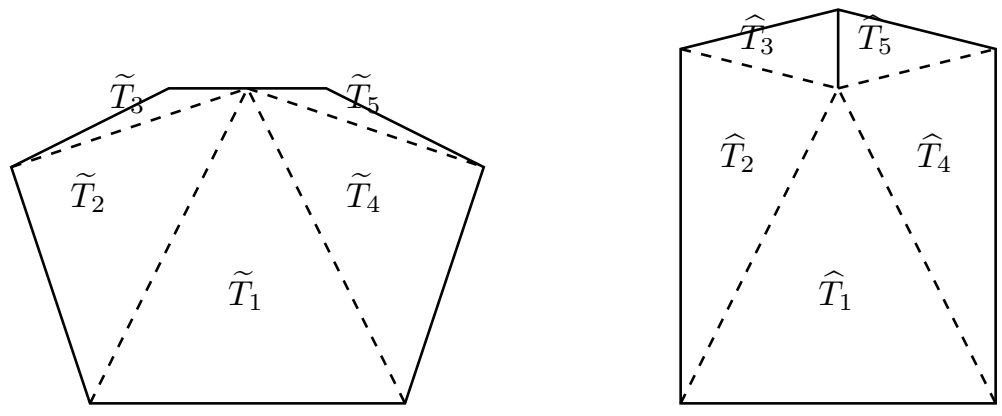

Figure 4: Left: the cell $\widetilde{Y}^{0}$. Right: the fractured open set $\widehat{Y}^{0}$. The Jacobian matrix of $H$ on the left (resp. of $H^{-1}$ on the right) jumps across the broken lines. In this figure, we have not respected the proportions (the areas of $\widetilde{T}_{i}$ and $\widehat{T}_{i}$ should coincide).

Note that for $i=1,2$, the abcissa of $G_{i}\left(x_{1}, x_{2}\right)$ only depends on $x_{1}$. This implies that for $i=1,2$, $G_{i}$ maps part of $\partial \widehat{Y}^{0}$ to vertical lines. Moreover, $G_{1}\left(P_{2}\right)=G_{2}\left(P_{1}\right)$ is a point on the axis $x_{1}=0$. We define

$$
\widehat{\mathcal{M}}_{\sigma}=G_{\sigma(1)} \circ \cdots \circ G_{\sigma(n)} \quad \text { for } \sigma \in \mathcal{A}_{n}
$$

the sets

$$
\widehat{Y}^{N}=\operatorname{Interior}\left(\widehat{\widehat{Y}}^{0} \cup \bigcup_{n=1}^{N} \bigcup_{\sigma \in \mathcal{A}_{n}} \widehat{\mathcal{M}}_{\sigma}\left(\overline{\widehat{Y}^{0}}\right)\right), \quad \widehat{\Omega}=\operatorname{Interior}\left(\overline{\widehat{Y}^{0}} \cup \bigcup_{n=1}^{\infty} \bigcup_{\sigma \in \mathcal{A}_{n}} \widehat{\mathcal{M}}_{\sigma}\left(\overline{\widehat{Y}^{0}}\right)\right),
$$

see Figure 5, and the one to one mapping

$$
\chi^{N}: \mid \begin{aligned}
\widehat{Y}^{N} & \rightarrow \widetilde{Y}^{N} \\
x & \mapsto \mathcal{M}_{\sigma} \circ H^{-1} \circ \widehat{\mathcal{M}}_{\sigma}^{-1}(x) \quad \text { if } x \in \widehat{\mathcal{M}}_{\sigma}\left(\widehat{Y}^{0}\right) .
\end{aligned}
$$

Note that $\chi^{N}$ is a piecewise affine map and that the Jacobian of $\chi^{N}$ is almost everywhere 1 . Moreover, take $\sigma \in \mathcal{A}_{n}$ with $n \leq N,\left(x_{1}, x_{2}\right) \in \widehat{\mathcal{M}}_{\sigma}\left(Y^{0}\right)$ and $h \in \mathbb{R}$ such that $\left(x_{1}, x_{2}+h\right) \in$ $\widehat{\mathcal{M}}_{\sigma}\left(Y^{0}\right)$. We aim at bounding $\left|\chi^{N}\left(x_{1}, x_{2}+h\right)-\chi^{N}\left(x_{1}, x_{2}\right)\right|$ : call $\left(z_{1}, z_{2}\right)=\widehat{\mathcal{M}}_{\sigma}^{-1}\left(x_{1}, x_{2}\right)$. It can be easily seen that $\widehat{\mathcal{M}}_{\sigma}^{-1}\left(x_{1}, x_{2}+h\right)=\left(z_{1}, z_{2}+\left(2 a^{2}\right)^{-n} h\right)$. Therefore,

$$
\begin{aligned}
\left|\chi^{N}\left(x_{1}, x_{2}+h\right)-\chi^{N}\left(x_{1}, x_{2}\right)\right| & =\left|\mathcal{M}_{\sigma} \circ H^{-1}\left(z_{1}, z_{2}+\left(2 a^{2}\right)^{-n} h\right)-\mathcal{M}_{\sigma} \circ H^{-1}\left(z_{1}, z_{2}\right)\right| \\
& \leq C_{H} a^{n}\left(2 a^{2}\right)^{-n}|h|=C_{H}(2 a)^{-n}|h|,
\end{aligned}
$$

where the constant $C_{H}$ is the norm of $H^{-1}$, and where we have used the fact that $\mathcal{M}_{\sigma}$ is a similitude with dilation ratio $a^{n}$. But $2 a>1$. Passing to the limit as $h$ tends to 0 , we see that

$$
\left\|\frac{\partial \chi^{N}}{\partial x_{2}}\right\|_{\infty} \leq C_{H}
$$

Note that $\widehat{Y^{N}}$ is contained in the rectangle $[-1,1] \times[0, \xi]$, where $\xi=r_{2} \sum_{n=0}^{\infty}\left(2 a^{2}\right)^{n}=\frac{r_{2}}{1-2 a^{2}}$. Moreover $\widehat{Y^{N}}$ has $I^{N}=2+\sum_{n=0}^{N} 2^{n}$ vertical boundaries, (among which $\sum_{n=0}^{N} 2^{n}$ vertical fractures) see Figure 5 . We order increasingly the abscissa $\left(\alpha_{i}\right)_{i=1, \ldots, I^{N}}$ of these vertical segments, and we have $\alpha_{i+1}-\alpha_{i}=2^{-N}, i=1, \ldots, I^{N}-1$. Notice also that $\widehat{Y}^{N}$ can be seen as the epigraph of a function $\Phi^{N}:(-1,1) \mapsto \mathbb{R}_{+}$, and that $\Phi^{N}$ is discontinuous at $\alpha_{i}, i=2, \ldots, I^{N}-1$, and linear in the intervals $\left(\alpha_{i}, \alpha_{i+1}\right), i=1, \ldots, I^{N}-1$. Another important and natural property is 


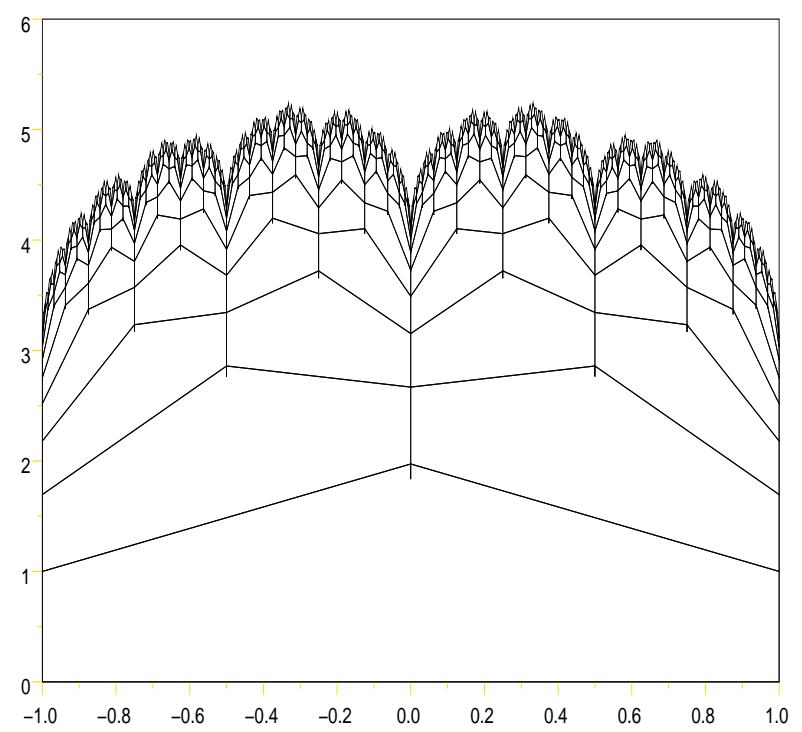

Figure 5: The open set $\widehat{\Omega}$ in the case when $\theta=\pi / 4, a=a^{*} \approx 0.593465, \alpha=1-a \cos \theta$ and $\beta=1+a \sin \theta$.

that the sequence $\left(\Phi^{N}\right)_{N}$ is nondecreasing with respect to $N$.

Call $\widehat{\Gamma}^{\infty}$ the self-similar set associated to $G_{1}$ and $G_{2}$, ( $\widehat{\Gamma}^{\infty}$ is part of the boundary of $\left.\widehat{\Omega}\right)$, and $\widehat{\mu}$ the self-similar measure associated to $\left(\widehat{\Gamma}^{\infty}, G_{1}, G_{2}\right)$. As above, it is possible to define a trace operator $\widehat{\ell}^{\infty}$ from $W^{1, q}(\widehat{\Omega})$ to $L^{q}\left(\widehat{\Gamma}^{\infty}\right)$.

Consider a function $v \in C^{\infty}(\Omega)$ such that $\left.v\right|_{\Gamma^{0}}=0$. Since $\chi^{N}$ is piecewise affine and continuous, and since $\left|\widetilde{\Gamma}^{N+1}\right|=2^{N+1}\left|\widetilde{\Gamma}^{\sigma}\right|$, for all $\sigma \in \mathcal{A}_{N+1}$,

$$
\frac{1}{\left|\widetilde{\Gamma}^{N+1}\right|} \int_{\widetilde{\Gamma}^{N+1}}|v|^{q}=\sum_{\sigma \in \mathcal{A}_{N+1}} \frac{1}{2^{N+1}\left|\widetilde{\Gamma}^{\sigma}\right|} \int_{\widetilde{\Gamma}^{\sigma}}|v|^{q}=\sum_{\sigma \in \mathcal{A}_{N+1}} \frac{1}{2^{N+1}\left|\widehat{\Gamma}^{\sigma}\right|} \int_{\widehat{\Gamma}^{\sigma}}\left|v \circ \chi^{N}\right|^{q} .
$$

On the other hand,

$$
\sum_{\sigma \in \mathcal{A}_{N+1}} \frac{1}{2^{N+1}\left|\widehat{\Gamma}^{\sigma}\right|} \int_{\widehat{\Gamma}^{\sigma}}\left|v \circ \chi^{N}\right|^{q}=\frac{1}{2} \sum_{i=0}^{I_{N}-1} \int_{\alpha_{i}}^{\alpha_{i+1}}\left|v \circ \chi^{N}\left(x, \Phi^{N}(x)\right)\right|^{q} d x .
$$

Therefore,

$$
\begin{aligned}
\frac{2}{\left|\widetilde{\Gamma}^{N+1}\right|} \int_{\widetilde{\Gamma}^{N+1}}|v|^{q} & =\sum_{i=0}^{I_{N}-1} \int_{\alpha_{i}}^{\alpha_{i+1}}\left|v\left(\chi^{N}\left(x, \Phi^{N}(x)\right)\right)\right|^{q} d x=\sum_{i=0}^{I_{N}-1} \int_{\alpha_{i}}^{\alpha_{i+1}}\left|\int_{0}^{\Phi^{N}(x)} \frac{d}{d t}\left(v\left(\chi^{N}(x, t)\right)\right) d t\right|^{q} d x \\
& =\sum_{i=0}^{I_{N}-1} \int_{\alpha_{i}}^{\alpha_{i+1}}\left|\sum_{j=0}^{N} \int_{\Phi^{j-1}(x)}^{\Phi^{j}(x)} \frac{d}{d t}\left(v\left(\chi^{N}(x, t)\right)\right) d t\right|^{q} d x
\end{aligned}
$$


Thus,

$$
\begin{aligned}
& \frac{2}{\left|\widetilde{\Gamma}^{N+1}\right|} \int_{\widetilde{\Gamma}^{N+1}}|v|^{q} \\
\leq & \sum_{i=0}^{I_{N}-1} \int_{\alpha_{i}}^{\alpha_{i+1}}\left(\sum_{j=0}^{N} \rho^{\frac{j}{q-1}}\right)^{q-1}\left(\sum_{j=0}^{N} \rho^{-j}\left|\int_{\Phi^{j-1}(x)}^{\Phi^{j}(x)} \frac{d}{d t}\left(v\left(\chi^{N}(x, t)\right)\right) d t\right|^{q}\right) d x \\
\lesssim & \sum_{i=0}^{I_{N}-1} \int_{\alpha_{i}}^{\alpha_{i+1}} \sum_{j=0}^{N} \rho^{-j}\left|\int_{\Phi^{j-1}(x)}^{\Phi^{j}(x)} \frac{d}{d t}\left(v\left(\chi^{N}(x, t)\right)\right) d t\right|^{q} d x \\
\leq & \sum_{i=0}^{I_{N}-1} \int_{\alpha_{i}}^{\alpha_{i+1}}\left(\sum_{j=0}^{N} \rho^{-j}\left(\Phi^{j}(x)-\Phi^{j-1}(x)\right)^{q-1} \int_{\Phi^{j-1}(x)}^{\Phi^{j}(x)} \mid \frac{d}{d t}\left(\left.v\left(\chi^{N}(x, t)\right)\right|^{q} d t\right) d x\right. \\
\lesssim & \sum_{i=0}^{I_{N}-1} \int_{\alpha_{i}}^{\alpha_{i+1}}\left(\sum_{j=0}^{N} \rho^{-j}\left(2 a^{2}\right)^{j(q-1)} \int_{\Phi^{j-1}(x)}^{\Phi^{j}(x)}\left|\frac{d}{d t}\left(v\left(\chi^{N}(x, t)\right)\right)\right|^{q} d t\right) d x
\end{aligned}
$$

where we have used several Hölder's inequalities. This yields that

$$
\begin{aligned}
& \frac{2}{\left|\widetilde{\Gamma}^{N+1}\right|} \int_{\widetilde{\Gamma}^{N+1}}|v|^{q} \\
\lesssim & \left(\begin{array}{l}
\sum_{i=0}^{I_{N}-1} \int_{\alpha_{i}}^{\alpha_{i+1}}\left(\sum_{j=0}^{N} \rho^{-j}\left(2 a^{2}\right)^{j(q-1)} \int_{\Phi^{j-1}(x)}^{\Phi^{j}(x)}\left|\frac{\partial v}{\partial x_{1}}\left(\chi^{N}(x, t)\right) \frac{\partial \chi_{1}^{N}}{\partial t}(x, t)\right|^{q} d t\right) d x \\
+\sum_{i=0}^{I_{N}-1} \int_{\alpha_{i}}^{\alpha_{i+1}}\left(\sum_{j=0}^{N} \rho^{-j}\left(2 a^{2}\right)^{j(q-1)} \int_{\Phi^{j-1}(x)}^{\Phi^{j}(x)}\left|\frac{\partial v}{\partial x_{2}}\left(\chi^{N}(x, t)\right) \frac{\partial \chi_{2}^{N}}{\partial t}(x, t)\right|^{q} d t\right) d x
\end{array}\right) .
\end{aligned}
$$

Using (44),

$$
\frac{1}{\left|\widetilde{\Gamma}^{N+1}\right|} \int_{\widetilde{\Gamma}^{N+1}}|v|^{q} \lesssim \sum_{i=0}^{I_{N}-1} \int_{\alpha_{i}}^{\alpha_{i+1}}\left(\sum_{j=0}^{N} \rho^{-j}\left(2 a^{2}\right)^{j(q-1)} \int_{\Phi^{j-1}(x)}^{\Phi^{j}(x)}\left|\nabla v\left(\chi^{N}(x, t)\right)\right|^{q} d t\right) d x .
$$

Performing the inverse change of variables, one obtains that

$$
\frac{1}{\left|\widetilde{\Gamma}^{N+1}\right|} \int_{\widetilde{\Gamma}^{N+1}}|v|^{q} \leq C\left(\int_{\widetilde{Y}^{0}}|\nabla v|^{q}+\sum_{n=1}^{N} \rho^{-n}\left(2 a^{2}\right)^{n(q-1)} \sum_{\sigma \in \mathcal{A}_{n}} \int_{\widetilde{\mathcal{M}}_{\sigma}\left(\widetilde{Y}^{0}\right)}|\nabla v|^{q}\right)
$$

where the constant $C$ can be chosen independently of $N$.

Finally, since $\frac{1}{\left|\widetilde{\Gamma}^{N+1}\right|} \int_{\widetilde{\Gamma}^{N+1}}|v|^{q}$ tends to $\int_{\widetilde{\Gamma}^{\infty}}\left|\widetilde{\ell}^{\infty}(v)\right|^{q} d \widetilde{\mu}$ as $N \rightarrow \infty$, we find that

$$
\int_{\widetilde{\Gamma}^{\infty}}\left|\widetilde{\ell}^{\infty}(v)\right|^{q} d \widetilde{\mu} \leq C\left(\int_{\widetilde{Y}^{0}}|\nabla v|^{q}+\sum_{n=1}^{\infty} \rho^{-n}\left(2 a^{2}\right)^{n(q-1)} \sum_{\sigma \in \mathcal{A}_{n}} \int_{\widetilde{\mathcal{M}}_{\sigma}\left(\widetilde{Y}^{0}\right)}|\nabla v|^{q}\right),
$$

which is the desired result. We conclude by using the density of $\left\{v \in \mathcal{C}^{\infty}(\Omega) ;\left.v\right|_{\Gamma^{0}}=0\right\}$ in $\mathcal{V}_{q}(\Omega)$. 
Step 2 We aim at proving (42) for $\widetilde{\Omega}$ by using Lemma 3 .

Let $V_{2}$ be the Banach space

$$
V_{2}=\left\{v: \widetilde{\Omega} \rightarrow \mathbb{R}^{2}, v \text { measurable }\|v\|_{L^{q}\left(\widetilde{Y}^{0}\right)}^{q}+\sum_{n=1}^{\infty} \rho^{-n}\left(2 a^{2}\right)^{n(q-1)} \sum_{\sigma \in \mathcal{A}_{n}}\|v\|_{L^{q}\left(\widetilde{\mathcal{M}}_{\sigma}\left(\widetilde{Y}^{0}\right)\right)}^{q}<\infty\right\},
$$

and let $V$ be the Banach space

$$
V=\left\{v \in L_{l o c}^{q}(\widetilde{\Omega}),\left.v\right|_{\widetilde{Y}^{0}} \in L^{q}\left(\widetilde{Y}^{0}\right), \nabla v \in V_{2}\right\},
$$

endowed with the norm

$$
\|v\|_{V}=\left(\left.\left|\int_{\widetilde{\Gamma}_{0}} v\right|_{\widetilde{\Gamma}^{0}}\right|^{q}+\|\nabla v\|_{L^{q}\left(\widetilde{Y}^{0}\right)}^{q}+\sum_{n=1}^{\infty} \rho^{-n}\left(2 a^{2}\right)^{n(q-1)} \sum_{\sigma \in \mathcal{A}_{n}}\|\nabla v\|_{L^{q}\left(\widetilde{\mathcal{M}}_{\sigma}\left(\widetilde{Y}^{0}\right)\right)}^{q}\right)^{1 / q} .
$$

Remark 10 It can be proved that

$$
V=\left\{v \in L^{q}(\widetilde{\Omega}), \nabla v \in V_{2}\right\} .
$$

It is clear from $(40)$ that $W^{1, q}(\widetilde{\Omega})$ is a subspace of $V$.

Furthermore, we will prove at the end of the paragraph that $W^{1, q}(\widetilde{\Omega})$ is dense in $V$.

As a consequence of $(41)$, one can extend the operator $\widetilde{\ell}^{\infty}$ to a continuous linear operator from $V$ to $W=L_{\widetilde{\mu}}^{q}=L^{q}\left(\widetilde{\Gamma}^{\infty}, d \widetilde{\mu}\right)$, that we still denote $\widetilde{\ell}^{\infty}$.

Let $\widetilde{P}_{0}$ be the projection on constants: $\widetilde{P}_{0} v=\int_{\widetilde{\Gamma}_{\infty}} v d \widetilde{\mu}$. It is straigthforward to see that the operator $L: v \mapsto \widetilde{\ell}^{\infty}(v)-\widetilde{P}_{0}\left(\widetilde{\ell}^{\infty}(v)\right)$ is also a continuous linear operator from $V$ to $W=L_{\widetilde{\mu}}^{q}$. Define $V_{1}$ as the space of the constant functions on $\widetilde{\Omega}$. We introduce the linear operators

$$
A_{1}: V \mapsto \mathbb{R}, \quad A_{1} v=\int_{\widetilde{\Gamma}_{0}} v, \quad \text { and } \quad A_{2}: V \mapsto V_{2}, \quad A_{2} v=\nabla v
$$

We can apply Lemma 3 since $\left.L\right|_{\text {ker } A_{2}} \equiv 0$. Therefore, there exists a constant $C>0$ such that $\left\|\widetilde{\ell}^{\infty}(v)-\widetilde{P}_{0}\left(\widetilde{\ell}^{\infty}(v)\right)\right\|_{L_{\widetilde{\mu}}^{q}}^{q} \leq C\left(\|\nabla v\|_{L^{q}\left(\widetilde{Y}^{0}\right)}^{q}+\sum_{n=1}^{\infty} \rho^{-n}\left(2 a^{2}\right)^{n(q-1)} \sum_{\sigma \in \mathcal{A}_{n}}\|\nabla v\|_{L^{q}\left(\widetilde{\mathcal{M}}_{\sigma}\left(\widetilde{Y}^{0}\right)\right)}^{q}\right), \quad \forall v \in V$,

which implies (42) in the case when $\Omega=\widetilde{\Omega}$, since $W^{1, q}(\widetilde{\Omega}) \subset V$.

Proof of the density of $W^{1, q}(\widetilde{\Omega})$ in $V$. We introduce a smooth real valued function $\phi$ defined on $\widetilde{Y}^{0}$ such that

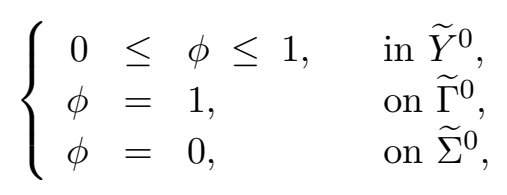

where

$$
\widetilde{\Sigma}^{0}=\left\{x \in \partial \widetilde{Y}^{0} \text { such that } x_{2}>b\right\} .
$$

For $n>0$ and $\sigma \in \mathcal{A}_{n}$, we also introduce the function $\phi_{\sigma}$ defined on $\widetilde{\mathcal{M}}_{\sigma}\left(\widetilde{Y}^{0}\right)$ by $\phi_{\sigma}=\phi \circ\left(\widetilde{\mathcal{M}}_{\sigma}\right)^{-1}$. For a function $v$ in $V$, we define $v_{n}$ by:

$$
\left\{\begin{aligned}
\left.v_{n}\right|_{\widetilde{Y}^{n-1}} & =\left.v\right|_{\widetilde{Y}^{n-1}}, \\
\left.v_{n}\right|_{\widetilde{\mathcal{M}}_{\sigma}\left(\widetilde{Y}^{0}\right)} & =\left.\phi_{\sigma} v\right|_{\widetilde{\mathcal{M}}_{\sigma}\left(\widetilde{Y}^{0}\right)}+\left(1-\phi_{\sigma}\right)\langle v\rangle_{\sigma}, \quad \forall \sigma \in \mathcal{A}_{n}, \\
\left.v_{n}\right|_{\widetilde{\Omega}^{\eta}} & =\langle v\rangle_{\sigma}, \quad \text { for } \quad \eta=\sigma \circ \widetilde{F}_{i}, \sigma \in \mathcal{A}_{n}, i=1,2,
\end{aligned}\right.
$$


where

$$
\langle v\rangle_{\sigma}=\frac{1}{\left|\widetilde{\Sigma}^{\sigma}\right|} \int_{\widetilde{\Sigma}^{\sigma}} v \quad \text { and } \quad \widetilde{\Sigma}^{\sigma}=\widetilde{\mathcal{M}}_{\sigma}\left(\widetilde{\Sigma}^{0}\right)
$$

It is easy to see that $u_{n} \in W^{1, q}(\widetilde{\Omega})$. Morover, $\lim _{n \rightarrow \infty}\left\|u-u_{n}\right\|_{V}=0$; indeed,

$$
\nabla u-\nabla u_{n}= \begin{cases}0 & \text { in } \widetilde{Y}^{n-1}, \\ \phi_{\sigma} \nabla u+\left(u-\langle u\rangle_{\sigma}\right) \nabla \phi_{\sigma} & \text { in } \widetilde{\mathcal{M}}_{\sigma}\left(\widetilde{Y}^{0}\right), \forall \sigma \in \mathcal{A}_{n} \\ \nabla u & \text { in } \widetilde{\Omega} \backslash \widetilde{Y}\end{cases}
$$

But $\left\|\nabla \phi_{\sigma}\right\|_{\infty}=a^{-n}\|\nabla \phi\|_{\infty}$. Therefore

$$
\begin{aligned}
\left\|u-u_{n}\right\|_{V}^{q} \leq c_{q}\left(\begin{array}{l}
\sum_{p \geq n} \rho^{-p}\left(2 a^{2}\right)^{p(q-1)} \sum_{\sigma \in \mathcal{A}_{p}} \int_{\widetilde{\mathcal{M}}_{\sigma}\left(\widetilde{Y}^{0}\right)}|\nabla u|^{q} \\
+\rho^{-n}\left(2 a^{2}\right)^{n(q-1)}\|\nabla \phi\|_{\infty}^{q} \sum_{\sigma \in \mathcal{A}_{n}} \int_{\widetilde{\mathcal{M}}_{\sigma}\left(\widetilde{Y}^{0}\right)} a^{-n q}\left|u-\langle u\rangle_{\sigma}\right|^{q}
\end{array}\right) \\
\leq c_{q}\left(\sum_{p \geq n} \rho^{-p}\left(2 a^{2}\right)^{p(q-1)} \sum_{\sigma \in \mathcal{A}_{p}} \int_{\widetilde{\mathcal{M}}_{\sigma}\left(\widetilde{Y}^{0}\right)}|\nabla u|^{q}+C_{q} \rho^{-p}\left(2 a^{2}\right)^{n(q-1)}\|\nabla \phi\|_{\infty}^{q} \int_{\widetilde{\mathcal{M}}_{\sigma}\left(\widetilde{Y}^{0}\right)}|\nabla u|^{q}\right),
\end{aligned}
$$

using the Poincaré-Wiertinger inequality in $\widetilde{\mathcal{M}}\left(\widetilde{Y}^{0}\right)$ :

$$
\int_{\widetilde{\mathcal{M}}\left(\widetilde{Y}^{0}\right)}|u-\langle u\rangle|^{q} \leq C_{q} a^{n q} \int_{\widetilde{\mathcal{M}}\left(\widetilde{Y}^{0}\right)}|\nabla u|^{q},
$$

and where the constants $c_{q}$ and $C_{q}$ do not depend on $n$. From this and Lebesgue theorem, we see that $\lim _{n \rightarrow \infty}\left\|u-u_{n}\right\|_{V}=0$.

Step 3 We now aim at proving (41) and (42) in the general case, i.e. for $Y^{0}$ satisfying the assumptions made in $\S 2.1 .3$. For that, we are going to construct a self-similar piecewise linear map from the domain $\Omega$ to $\widetilde{\Omega}$. It is easy to see that there exist

1. a partition $\mathcal{T}$ of $Y^{0}$ into $p$ non-overlapping triangles, such that $\Gamma^{0}, F_{1}\left(\Gamma^{0}\right), F_{2}\left(\Gamma^{0}\right)$ are the whole side of a triangle in the partition.

2. a partition $\widetilde{\mathcal{T}}$ of $\widetilde{Y}^{0}$ into $p$ non-overlapping triangles, such that $\widetilde{\Gamma}^{0}, \widetilde{F}_{1}\left(\widetilde{\Gamma}^{0}\right), \widetilde{F}_{2}\left(\widetilde{\Gamma}^{0}\right)$ are the whole side of a triangle in the partition.

3. a continuous, one to one and piecewise linear function $\psi$ from $Y^{0}$ onto $\tilde{Y}^{0}$, such that

- its restriction to the triangles in $\mathcal{T}$ is linear,

- $\psi$ maps each triangle in $\mathcal{T}$ to a triangle in $\tilde{\mathcal{T}}$,

- the restriction of $\psi$ to $\Gamma^{0}$ is the identity,

- for $i=1,2$,

$$
F_{i}\left(\psi^{-1}(x)\right)=\psi^{-1}\left(\widetilde{F}_{i}(x)\right), \quad \forall x \in \widetilde{\Gamma}^{0} .
$$

An example of such a construction is shown in Figure 6.

This construction allows for the definition of the continuous linear operator

$$
\Psi: W^{1, q}(\Omega) \rightarrow W^{1, q}(\widetilde{\Omega}),
$$




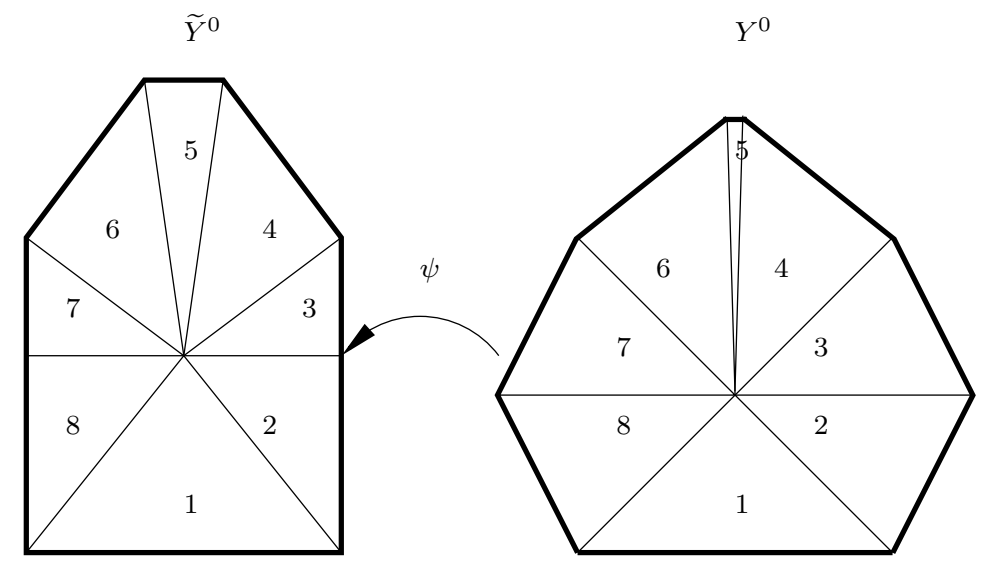

Figure 6: The domains $Y^{0}$ and $\widetilde{Y}^{0}$ are partitioned into eight triangles

$\Psi(u)=\widetilde{u}, \quad \widetilde{u}$ defined by $\left\{\begin{aligned}\left.\widetilde{u}\right|_{\widetilde{Y}^{0}} & =\left.u\right|_{Y^{0}} \circ \psi^{-1}, \\ \left.\widetilde{u}\right|_{\widetilde{\mathcal{M}}_{\sigma}\left(\widetilde{Y}^{0}\right)} & =\left.u\right|_{\mathcal{M}_{\sigma}\left(Y^{0}\right)} \circ \mathcal{M}_{\sigma} \circ \psi^{-1} \circ\left(\widetilde{\mathcal{M}}_{\sigma}\right)^{-1}, \quad \sigma \in \mathcal{A}_{n}, n \geq 1 .\end{aligned}\right.$

Using the same argument as in the proof of Theorem 4 in [2], we have that

$$
\left\|\ell^{\infty}(v)\right\|_{L_{\mu}^{q}} \lesssim\left\|\tilde{\ell}^{\infty}(\Psi(v))\right\|_{L_{\tilde{\mu}}^{q}}, \quad \forall v \in \mathcal{V}_{q}(\Omega)
$$

From (48) and (45), we see that

$$
\left\|\ell^{\infty}(v)\right\|_{L_{\mu}^{q}}^{q} \lesssim \int_{\widetilde{Y}^{0}}|\nabla(\Psi(v))|^{q}+\sum_{n=1}^{\infty} \rho^{-n}\left(2 a^{2}\right)^{n(q-1)} \sum_{\sigma \in \mathcal{A}_{n}} \int_{\widetilde{\mathcal{M}}_{\sigma}\left(\widetilde{Y}^{0}\right)}|\nabla(\Psi(v))|^{q}, \quad \forall v \in \mathcal{V}_{q}(\Omega) .
$$

The inverse change of variables yields (41).

Similarly, the proof of (42) follows from the observation that $P_{0}\left(\ell^{\infty}(v)\right)=\widetilde{P}_{0}\left(\widetilde{\ell}^{\infty}(\Psi(v))\right)$ and from (46).

The case $a=1 / 2$. See Appendix B.

Proof of Theorem 10 Call $f=\ell^{\infty}(v)$. Recall that

$$
\|f\|_{J \operatorname{Lip}\left(1-\frac{2-d}{q}, q, q ; 0 ; \Gamma^{\infty}\right)}^{q}=\|f\|_{L_{\mu}^{q}}^{q}+\int_{\Gamma^{\infty}}\left|f-P_{0} f\right|^{q} d \mu+\sum_{n=1}^{\infty}\left(2 a^{2-q}\right)^{n} \sum_{\sigma \in \mathcal{A}_{n}} \int_{\mathcal{M}_{\sigma}\left(\Gamma^{\infty}\right)}\left|f-P_{\sigma} f\right|^{q} d \mu .
$$

We have to prove that the right side of (50) is finite. A change of variables leads to

$$
\begin{aligned}
& \sum_{n=1}^{\infty}\left(2 a^{2-q}\right)^{n} \sum_{\sigma \in \mathcal{A}_{n}} \int_{\mathcal{M}_{\sigma}\left(\Gamma^{\infty}\right)}\left|f-P_{\sigma} f\right|^{q} d \mu \\
= & \sum_{n=1}^{\infty}\left(2 a^{2-q}\right)^{n} \sum_{\sigma \in \mathcal{A}_{n}} 2^{-n} \int_{\Gamma^{\infty}}\left|f \circ \mathcal{M}_{\sigma}-P_{0}\left(f \circ \mathcal{M}_{\sigma}\right)\right|^{q} d \mu .
\end{aligned}
$$


This and Theorem 11 imply that

$$
\begin{aligned}
& \sum_{n=1}^{\infty}\left(2 a^{2-q}\right)^{n} \sum_{\sigma \in \mathcal{A}_{n}} \int_{\mathcal{M}_{\sigma}\left(\Gamma^{\infty}\right)}\left|f-P_{\sigma} f\right|^{q} d \mu \\
\lesssim & \sum_{n=1}^{\infty} a^{n(2-q)} \sum_{\sigma \in \mathcal{A}_{n}}\left(\left\|\nabla\left(v \circ \mathcal{M}_{\sigma}\right)\right\|_{L^{q}\left(Y^{0}\right)}^{q}+\sum_{p=1}^{\infty} \rho^{-p}\left(2 a^{2}\right)^{p(q-1)} \sum_{\eta \in \mathcal{A}_{p}}\left\|\nabla\left(v \circ \mathcal{M}_{\sigma}\right)\right\|_{L^{q}\left(\mathcal{M}_{\eta}\left(Y^{0}\right)\right)}^{q}\right) \\
= & \sum_{n=1}^{\infty} a^{n(2-q)} \sum_{\sigma \in \mathcal{A}_{n}}\left(a^{n(q-2)}\left(\|\nabla v\|_{L^{q}\left(\mathcal{M}_{\sigma}\left(Y^{0}\right)\right)}^{q}+\sum_{p=1}^{\infty} \rho^{-p}\left(2 a^{2}\right)^{p(q-1)} \sum_{\eta \in \mathcal{A}_{p}}\|\nabla v\|_{L^{q}\left(\mathcal{M}_{\sigma \circ \eta}\left(Y^{0}\right)\right)}^{q}\right)\right)
\end{aligned}
$$

where $\rho$ is such that $\left(2 a^{2}\right)^{q-1}<\rho<1$. Thus,

$$
\sum_{n=1}^{\infty}\left(2 a^{2-q}\right)^{n} \sum_{\sigma \in \mathcal{A}_{n}} \int_{\mathcal{M}_{\sigma}\left(\Gamma^{\infty}\right)}\left|f-P_{\sigma} f\right|^{q} d \mu \lesssim \sum_{i=1}^{\infty} \rho^{-i}\left(2 a^{2}\right)^{i(q-1)}\left(\sum_{n=1}^{i} \rho^{n}\left(2 a^{2}\right)^{n(1-q)}\right) \sum_{\mu \in \mathcal{A}_{i}}\|\nabla v\|_{L^{q}\left(\mathcal{M}_{\mu}\left(Y^{0}\right)\right)}^{q} .
$$

From $\rho\left(2 a^{2}\right)^{1-q}>1$, we deduce that $\sum_{n=1}^{i} \rho^{n}\left(2 a^{2}\right)^{n(1-q)} \lesssim \rho^{i}\left(2 a^{2}\right)^{i(1-q)}$. Thus,

$$
\sum_{n=1}^{\infty}\left(2 a^{2-q}\right)^{n} \sum_{\sigma \in \mathcal{A}_{n}} \int_{\mathcal{M}_{\sigma}\left(\Gamma^{\infty}\right)}\left|f-P_{\sigma} f\right|^{q} d \mu \lesssim \sum_{i=1}^{\infty} \sum_{\mu \in \mathcal{A}_{i}}\|\nabla v\|_{L^{q}\left(\mathcal{M}_{\mu}\left(Y^{0}\right)\right)}^{q},
$$

and the desired result follows easily.

\section{Traces of functions belonging to $W^{m+1, q}(\Omega)$ for a positive inte- ger $m$.}

We now assume that $a \geq 1 / 2$ and that $\Gamma^{\infty}$ is not contained in a straight line. We use the notations defined in $\S 4.2$. To keep the length of the paper reasonable, some proofs will be omitted or just sketched. For any real number $q \geq 1$ and any nonnegative integer $m$, the space $W^{m+1, q}(\Omega)$ is the space of the functions in $L^{q}(\Omega)$ such that all their partial derivatives of order less than or equal to $m+1$ (in the sense of distribution) belong to $L^{q}(\Omega)$.

Proposition 6 For all real number $q \geq 1$, there exists a constant $C$ such that for all $f \in$ $J \operatorname{Lip}\left(m+1-\frac{2-d}{q}, q, q ; m ; \Gamma^{\infty}\right)$, there exists $\tilde{f} \in W^{m+1, q}(\Omega)$ with the properties $\left.\tilde{f}\right|_{Y^{0}}=0$, $\ell^{\infty}(\tilde{f})=f$ and

$$
\|\tilde{f}\|_{W^{m+1, q}(\Omega)}^{q} \approx \int_{\Omega}\left|D^{m+1} \tilde{f}\right|^{q} \leq C\|f\|_{J L i p\left(m+1-\frac{2-d}{q}, q, q ; m ; \Gamma^{\infty}\right)}^{q} .
$$

Proof. The proof resembles very much that of Proposition 5. It mainly consists of constructing functions $\tilde{g}^{0, i} \in W^{m+1, q}(\Omega), i=1, \ldots M_{0}$ such that

- $\left.\tilde{g}^{0, i}\right|_{Y^{0}}=0$,

- $\left.\tilde{g}^{0, i}\right|_{\mathcal{M}_{\sigma}(\Omega)}$ belongs to $\mathcal{P}_{m}$, for all $\sigma \in \mathcal{A}_{2}$,

- $\ell^{\infty}\left(\tilde{g}^{0, i}\right)=g^{0, i}$. 
For a positive integer $n$ and $\sigma \in \mathcal{A}_{n}$, let us define the function $\tilde{g}^{\sigma, i}$ by:

$$
\begin{aligned}
\left.\tilde{g}^{\sigma, i}\right|_{\Omega^{\sigma}} & =2^{n / 2} \tilde{g}^{0, i} \circ \mathcal{M}_{\sigma}^{-1}, \\
\left.\tilde{g}^{\sigma, i}\right|_{\Omega \backslash \Omega^{\sigma}} & =0 .
\end{aligned}
$$

From the definition of $g^{\sigma, i}$, we see that $\ell^{\infty}\left(\tilde{g}^{\sigma, i}\right)=g^{\sigma, i}$. For a fixed $i \in\left\{1, \ldots, M_{0}\right\}$,

- the supports of $D^{m+1} \tilde{g}^{\sigma, i}$ and $D^{m+1} \tilde{g}^{0, i}$ are disjoint,

- if $(n, \sigma) \neq\left(n^{\prime}, \eta\right)$ then the supports of $D^{m+1} \tilde{g}^{\sigma, i}$ and $D^{m+1} \tilde{g}^{\eta, i}$ are disjoint.

Consider also $M_{0}$ functions $\tilde{\phi}_{i} \in W^{m+1, q}(\Omega)$ such that

- $\left.\tilde{\phi}^{i}\right|_{Y^{0}}=0$,

- $\ell^{\infty}\left(\tilde{\phi}^{i}\right)=\phi^{i}$.

For a funtion $f \in J \operatorname{Lip}\left(m+1-(2-d) / q, q, q ; m ; \Gamma^{\infty}\right)$ given by the expansion (28), it can be shown as in the proof of Proposition 5 that the function $\tilde{f}$ given by

$$
\tilde{f}=\sum_{i=1}^{M_{0}} \lambda_{i} \tilde{\phi}^{i}+\sum_{i=1}^{M_{0}} \beta_{0, i} \tilde{g}^{0, i}+\sum_{i=1}^{M_{0}} \sum_{n \geq 1} \sum_{\sigma \in \mathcal{A}_{n}} \beta_{n, \sigma, i} \tilde{g}^{\sigma, i}
$$

has the desired properties.

We have the analogue of Theorem 11:

Theorem 12 For all $q, 1<q<\infty$ and all real number $\rho$ satisfying (40), there exists a constant $C$ such that, for all $v \in W^{1, q}(\Omega)$,

$$
\left\|\ell^{\infty}(v)-P_{0}^{(m)}\left(\ell^{\infty}(v)\right)\right\|_{L_{\mu}^{q}}^{q} \leq C\left(\begin{array}{l}
\left\|D^{m+1} v\right\|_{L^{q}\left(Y^{0}\right)}^{q} \\
+\sum_{n=1}^{\infty} \rho^{-n(m+1)}\left(2 a^{2}\right)^{n((m+1) q-1)} \sum_{\sigma \in \mathcal{A}_{n}}\left\|D^{m+1} v\right\|_{L^{q}\left(\mathcal{M}_{\sigma}\left(Y^{0}\right)\right)}^{q}
\end{array}\right) .
$$

Proof. We skip the proof, because it is long and resembles that of Theorem 11.

Theorem 12 makes it possible to prove the analogue of Theorem 10:

Theorem 13 For all $q, 1<q<\infty$ and $v \in W^{m+1, q}(\Omega)$, $\ell^{\infty}(v)$ belongs to JLip $(m+1-$ $\left.\frac{2-d}{q}, q, q ; m ; \Gamma^{\infty}\right)$ and there exists a constant $c$ such that

$$
\left\|\ell^{\infty}(v)\right\|_{J L i p\left(m+1-\frac{2-d}{q}, q, q ; m ; \Gamma^{\infty}\right)} \leq c\|v\|_{W^{m+1, q}(\Omega)}, \quad \forall v \in W^{m+1, q}(\Omega) .
$$

Proof. The proof is very similar to that of Theorem 10 .

From Proposition 6 and Theorem 13, we obtain that for all $q, 1<q<\infty$,

$$
\ell^{\infty}\left(W^{m+1, q}(\Omega)\right)=J \operatorname{Lip}\left(m+1-\frac{2-d}{q}, q, q ; m ; \Gamma^{\infty}\right) .
$$




\section{Appendix}

\section{A Sketched proofs of Theorems 2 and 3}

\section{A.1 Preliminary lemmas}

Lemma 4 For $0<\theta<\pi / 2$, assume that $(\alpha, \beta)$ satisfy (5) for $a=a^{*}$ and (6). Then, for all $a$, $0 \leq a<a^{*}$, the convex hull of $\left(\overline{\widetilde{Y}^{0}} \cup\left(\bigcup_{n=1}^{N} \underset{\sigma \in \mathcal{A}_{n}}{\cup} \mathcal{M}_{\sigma}\left(\overline{\widetilde{Y}^{0}}\right)\right)\right)$ coincides with the convex hull of $\left\{P_{1}, P_{2}\right\} \cup\left(\underset{\sigma \in \mathcal{A}_{N+1}}{\cup}\left\{\mathcal{M}_{\sigma}\left(P_{1}\right), \mathcal{M}_{\sigma}\left(P_{2}\right)\right\}\right)$.

Proof. First step Call $\chi^{N}$ the convex hull of $\overline{\widetilde{Y}^{0}} \cup\left(\bigcup_{n=1}^{N} \cup \underset{\sigma \in \mathcal{A}_{n}}{ } \mathcal{M}_{\sigma}\left(\overline{\widetilde{Y}^{0}}\right)\right)$.

We first observe that the points $F_{1}\left(P_{1}\right), F_{1}\left(P_{2}\right), F_{2}\left(P_{1}\right)$, and $F_{2}\left(P_{2}\right)$ are strictly contained in $\chi^{1}$ : this can be checked by simple geometric arguments: for example, one sees that the angle between the line segments $\left[P_{2}, F_{2}\left(P_{2}\right)\right]$ and $F_{2}\left(\left[P_{2}, F_{2}\left(P_{2}\right)\right]\right)$ is $\pi-\theta<\pi$, from which we deduce that $F_{2}\left(P_{2}\right)$ is strictly contained in $\chi^{1}$. From this, we deduce that the points $F_{1}\left(P_{1}\right), F_{1}\left(P_{2}\right)$, $F_{2}\left(P_{1}\right)$, and $F_{2}\left(P_{2}\right)$ are strictly contained in $\chi^{N}$ for all $N \geq 1$, because $\chi^{1} \subset \chi^{N}$.

Second step We prove Lemma 4 by induction. We have just seen that the claim is true for $N=1$. Assume that the claim is true up to $N$. We have

$$
\chi^{N+1}=\text { convex hull }\left(\overline{\widetilde{Y}^{0}} \cup F_{1}\left(\chi^{N}\right) \cup F_{2}\left(\chi^{N}\right)\right) .
$$

From the induction hypothesis, we deduce

$$
\chi^{N+1}=\text { convex hull }\left(\left\{P_{0}, P_{1}, F_{1}\left(P_{1}\right), F_{1}\left(P_{2}\right), F_{2}\left(P_{1}\right), F_{2}\left(P_{2}\right)\right\} \cup\left(\underset{\sigma \in \mathcal{A}_{N+2}}{\cup}\left\{\mathcal{M}_{\sigma}\left(P_{1}\right), \mathcal{M}_{\sigma}\left(P_{2}\right)\right\}\right)\right) .
$$

But we know that $F_{1}\left(P_{1}\right), F_{1}\left(P_{2}\right), F_{2}\left(P_{1}\right)$, and $F_{2}\left(P_{2}\right)$ are strictly contained in $\chi^{N+1}$ : therefore,

$$
\chi^{N+1}=\text { convex hull }\left(\left\{P_{0}, P_{1}\right\} \cup \underset{\sigma \in \mathcal{A}_{N+2}}{\cup}\left\{\mathcal{M}_{\sigma}\left(P_{1}\right), \mathcal{M}_{\sigma}\left(P_{2}\right)\right\}\right) .
$$

By passing to the limit as $N \rightarrow \infty$, we obtain that:

Lemma 5 Assume that $(\alpha, \beta)$ satisfy (5) for $a=a^{*}$ and (6). Then, for all $a, 0 \leq a<a^{*}$, the convex hull of $\widetilde{\widetilde{\Omega}}$ is the convex hull of $\left\{P_{1}, P_{2}\right\} \cup \Gamma^{\infty}$.

\section{A.2 Proof of Theorem 2}

With an argument similar to those used in [16] and elementary calculus, one can prove:

Lemma 6 For $\theta, 0<\theta<\pi / 2$, call $\left(F_{2} \circ F_{1}\right)^{\infty}$ the fixed point of $F_{2} \circ F_{1}$ and $\zeta$ the smallest integer such that $\zeta \theta \geq \pi$. The point $F_{1}^{\zeta}\left(F_{2} \circ F_{1}\right)^{\infty}$ is a point in $\Gamma^{\infty}$ with minimal ordinate. Its ordinate $Z$ takes the value

$$
Z=\beta-\alpha \frac{\cos \theta}{\sin \theta}+\frac{1}{1-a^{2}}\left(\beta a-\frac{\alpha(1-a \cos \theta)}{\sin \theta}\right)\left(\sum_{i=1}^{\zeta} a^{i-1} \cos i \theta-a^{2} \sum_{i=1}^{\zeta-2} a^{i-1} \cos i \theta\right) .
$$


Lemma 7 For $\theta, 0<\theta<\pi / 2$, for any $\alpha>a^{*} \cos \theta$, there exists $\bar{\beta}>0$ such that $\bar{\beta}>a^{*} \sin \theta$ and $(\alpha-1) \sin \theta+\bar{\beta} \cos \theta \geq 0$ and for any $\beta \geq \bar{\beta}$, $\Gamma^{\infty}$ is contained in the half-plane $\left\{x: x_{2}>0\right\}$ for all $a, 0<a \leq a^{*}$.

Proof. From (57), we see that $Z$ is affine with respect to $\beta$. Thus, Lemma 7 will be proved if show that for a positive constant $c$ depending on $\theta$ but not on $a, 0 \leq a<a^{*}$,

$$
1+\frac{a}{1-a^{2}}\left(\sum_{i=1}^{\zeta} a^{i-1} \cos i \theta-a^{2} \sum_{i=1}^{\zeta-2} a^{i-1} \cos i \theta\right) \geq c,
$$

or in an equivalent manner

$$
\frac{1}{1-a^{2}}\left(\sum_{i=0}^{\zeta} a^{i} \cos i \theta-\sum_{i=0}^{\zeta-2} a^{i+2} \cos i \theta\right) \geq c .
$$

Since $a^{*}$ is the positive solution of (4), $0 \leq a<a^{*}$ implies that $-\sum_{i=0}^{\kappa-1} a^{i+2} \cos i \theta \geq-1 / 2$. Since $\zeta \geq \kappa+1$, and since $a^{*} \leq 1 / \sqrt{2}$, we see that a sufficient condition for (58) is

$$
\frac{1}{2}+\sum_{i=1}^{\zeta} a^{i} \cos i \theta-a^{2} \sum_{i=\kappa}^{\zeta-2} a^{i} \cos i \theta \geq c,
$$

for a different constant $c$. We make out two cases:

a) the case when $(2 \kappa-1)<\pi$ which implies $\zeta=2 \kappa$ and $\theta<\pi / 3$; b) the case when $(2 \kappa-1) \theta \geq \pi$ which implies $\zeta=2 \kappa-1$; for technical reasons, we split the case b) into two subcases: b1) $\kappa=2$ and $\zeta=3$, which occurs for $\pi / 3 \leq \theta<\pi / 2$; b2) $\kappa \geq 3$ so $\theta<\pi / 4$.

a) $(2 \kappa-1) \theta<\pi$. In this case, $0<\theta<\pi / 3$ and $\zeta=2 \kappa$. We have

$$
\frac{1}{2}+\sum_{i=1}^{\zeta} a^{i} \cos i \theta-a^{2} \sum_{i=\kappa}^{\zeta-2} a^{i} \cos i \theta=T_{0}+\sum_{i=1}^{\kappa-1} T_{i}+S,
$$

where $S=-a^{2} \sum_{i=\kappa}^{\zeta-2} a^{i} \cos i \theta \geq 0, T_{i}=a^{i} \cos i \theta+a^{2 \kappa-i-1} \cos ((2 \kappa-i-1) \theta), i=1, \ldots, \kappa-1$, and $T_{0}=1 / 2+a^{2 \kappa-1} \cos ((2 \kappa-1) \theta)+a^{2 \kappa} \cos (2 \kappa \theta)$. One sees that for $i=1, \ldots, \kappa-1,-\cos ((2 \kappa-$ $i-1) \theta) \leq \cos i \theta$, so $T_{i} \geq 0$. On the other hand $T_{0} \geq 1 / 2-a^{2 \kappa-1}-a^{2 \kappa} \geq 1 / 2-\left(a^{*}\right)^{3}-\left(a^{*}\right)^{4}$ and since $a^{*}(\theta)<a^{*}(\pi / 3) \sim 0.62,1 / 2-\left(a^{*}\right)^{3}-\left(a^{*}\right)^{4} \geq c>0$.

b1) $\kappa=2$ and $\zeta=3$. In this case, $\pi / 3 \leq \theta<\pi / 2$ and the left hand side of (59) takes the simple form $1 / 2\left(1+2 a \cos \theta+2 a^{2} \cos 2 \theta+2 a^{3} \cos 3 \theta\right)$. We have

$$
\begin{aligned}
1+2 a \cos \theta+2 a^{2} \cos 2 \theta+2 a^{3} \cos 3 \theta & =1+2 a \cos \theta+2 a^{2} \cos 2 \theta+2 a^{3}(\cos \theta \cos 2 \theta-\sin \theta \sin 2 \theta) \\
& \geq 1+2 a \cos \theta+2 a^{2} \cos 2 \theta+\left(1-2 a^{2}\right) \cos 2 \theta-2 a^{3} \sin \theta \sin 2 \theta,
\end{aligned}
$$

where the latter estimate comes from the fact that $a \leq a^{*}, 2\left(a^{*}\right)^{3} \cos \theta=1-2\left(a^{*}\right)^{2}$ and $\cos 2 \theta<0$. Thus,

$$
\begin{aligned}
1+2 a \cos \theta+2 a^{2} \cos 2 \theta+2 a^{3} \cos 3 \theta & \geq 1+2 a \cos \theta+\cos 2 \theta-2 a^{3} \sin \theta \sin 2 \theta \\
& =\cos \theta\left(2 \cos \theta+2 a-4 a^{3} \sin ^{2} \theta\right) \\
& =\cos \theta\left(2 \cos \theta+2 a\left(1-2 a^{2}\right)+4 a^{3} \cos ^{2} \theta\right) \geq c>0,
\end{aligned}
$$

because $a \leq a^{*}<1 / \sqrt{2}$. 
b2) $\zeta=2 \kappa-1$ and $\kappa \geq 3$. In this case, $0<\theta<\pi / 4$

$$
\frac{1}{2}+\sum_{i=1}^{\zeta} a^{i} \cos i \theta-a^{2} \sum_{i=\kappa}^{\zeta-2} a^{i} \cos i \theta=T_{0}+\sum_{i=1}^{\kappa-1} T_{i}+S,
$$

where $S=a^{\kappa-1} \cos ((\kappa-1) \theta)-a^{2} \sum_{i=\kappa}^{\zeta-2} a^{i} \cos i \theta \geq 0, T_{i}=a^{i} \cos i \theta+a^{2 \kappa-i-2} \cos ((2 \kappa-i-2) \theta)$, $i=1, \ldots, \kappa-2$, and $T_{0}=1 / 2+a^{2 \kappa-2} \cos ((2 \kappa-2) \theta)+a^{2 \kappa-1} \cos ((2 \kappa-1) \theta)$. One sees that for $i=1, \ldots, \kappa-2,-\cos ((2 \kappa-i-2) \theta) \leq \cos i \theta$, so $T_{i} \geq 0$. On the other hand $T_{0} \geq$ $1 / 2-a^{2 \kappa-2}-a^{2 \kappa-1} \geq 1 / 2-\left(a^{*}\right)^{4}-\left(a^{*}\right)^{5}$ and the latter is greater than $c>0$.

Lemma 8 For $\theta, 0<\theta<\pi / 2$, for any $\alpha>a^{*} \cos \theta$, there exists $\bar{\beta}>0$ such that $\bar{\beta}>a \sin \theta$ and $(\alpha-1) \sin \theta+\bar{\beta} \cos \theta \geq 0$ and for any $\beta \geq \bar{\beta}$, $\widetilde{\Omega}$ is contained in the half-plane $\left\{x: x_{2}>0\right\}$ for all $a, 0<a \leq a^{*}$.

Proof. This is a consequence of Lemmas 7 and 5 .

Proof of Theorem 2 Take $a \leq a^{*}$ : from Lemma 8, $\widetilde{\Omega}$ lies in the half-plane $\left\{x: x_{2}>0\right\}$; by similarity, $F_{1}(\widetilde{\Omega})$ is contained in the open half-plane above the straight line joining $F_{1}\left(P_{1}\right)$ and $F_{1}\left(P_{2}\right)$. Therefore $F_{1}(\widetilde{\Omega}) \cap \widetilde{Y}^{0}=\emptyset$. Similarly, $F_{2}(\widetilde{\Omega}) \cap \widetilde{Y}^{0}=\emptyset$.

Moreover for $a<a^{*}, F_{1}\left(\Gamma^{\infty}\right)$ is contained in the half-plane $\left\{x: x_{1}<0\right\}$ otherwise, by symmetry, $F_{1}\left(\Gamma^{\infty}\right) \cap F_{2}\left(\Gamma^{\infty}\right)$ would not be empty. By continuity, $F_{1}\left(\Gamma^{\infty}\right)$ is contained in the half-plane $\left\{x: x_{1} \leq 0\right\}$ for $a=a^{*}$. This implies by Lemma 5 and self-similarity that $F_{1}(\widetilde{\Omega})$ is contained in the open half-space $\left\{x: x_{1}<0\right\}$ for $a \leq a^{*}$. Similarly, $F_{2}(\widetilde{\Omega})$ is contained in the open half-space $\left\{x: x_{1}>0\right\}$ for $a \leq a^{*}$. Therefore $F_{1}(\widetilde{\Omega}) \cap F_{2}(\widetilde{\Omega})=\emptyset$ for $a \leq a^{*}$.

Collecting the above results and using self-similarity, it is easy to check that $(\alpha, \beta)$ satisfies Assumption 1.

\section{A.3 Proof of Theorem 3}

We define some useful notations: we note $L$ the axis $\left\{x: x_{1}=0\right\}$. By symmetry, the intersections of the lines $L, F_{1}(L)$ and $F_{2}(L)$ is a point called $A$, see Figure 7 . We call $L_{1}^{\prime}$ (resp. $L_{2}^{\prime}$ ) the symmetric of $L$ with respect to $F_{1}(L)$ (resp. $F_{2}(L)$ ). We call $S_{1}$ (resp. $S_{2}$ ) the sector limited by the lines $L$ and $L_{1}^{\prime}\left(\operatorname{resp} L\right.$ and $\left.L_{2}^{\prime}\right)$ : the vertex of $S_{1}$ is $A, S_{1}$ is symmetric with respect to $F_{1}(L)$ and the aperture of $S_{1}$ is $2 \theta$. We introduce $S=S_{1} \cup S_{2}$ the sector of vertex $A$, of aperture $4 \theta$ which is symmetric w.r.t. $L$, see Figure 7.

\section{A.3.1 The case $\pi / 6 \leq \theta \leq \pi / 4$}

If $\pi / 6 \leq \theta \leq \pi / 4, a^{*}$ is the unique positive solution of the equation

$$
-1+2 X^{2}+2 \cos \theta X^{3}+2 \cos 2 \theta X^{4}=0 .
$$

Lemma 9 If $F_{1}$ and $F_{2}$ are given by (1) with $\alpha=a \cos \theta$ and $\beta=a \sin \theta, \pi / 6 \leq \theta \leq \pi / 4$, then for all $a, 0<a<a^{*}, \Gamma^{\infty}$ is contained in the half-plane $\left\{x: x_{2}>0\right\}$.

Proof. (sketched) Here, $A=\left(0,-a \frac{\cos 2 \theta}{\sin \theta}\right)$. Since $\Gamma^{\infty}$ is symmetric with respect to $L, F_{1}\left(\Gamma^{\infty}\right)$ is symmetric with respect to $F_{1}(L)$. Similarly $F_{2}\left(\Gamma^{\infty}\right)$ is symmetric with respect to $F_{2}(L)$. For $a<a^{*}, F_{1}\left(\Gamma^{\infty}\right)$ lies strictly on side of the axis $L$ (because $F_{1}\left(\Gamma^{\infty}\right) \cap F_{2}\left(\Gamma^{\infty}\right)=\emptyset$ ). By symmetry with respect to $F_{1}(L), F_{1}\left(\Gamma^{\infty}\right)$ is contained in the sector $S_{1}$. Similarly, $F_{2}\left(\Gamma^{\infty}\right)$ is contained in the sector $S_{2}$. Therefore $\Gamma^{\infty} \subset S=S_{1} \cup S_{2}$.

If $\theta=\pi / 4, S$ is the half-plane $\left\{x: x_{2}>0\right\}$ and the proof is finished. 
If $\pi / 6 \leq \theta<\pi / 4$, by similarity, $F_{1}\left(\Gamma^{\infty}\right)$ is contained in $S_{1} \cap F_{1}(S)$. Easy, but long calculations using (60) show that $S_{1} \cap F_{1}(S)$ is contained in the half-plane $\left\{x: x_{2}>0\right\}$, see Figure 7 . We do not write them explicitly. Similarly $F_{2}\left(\Gamma^{\infty}\right)$ is contained in $S_{1} \cap F_{2}(S)$, which is also contained in $\left\{x: x_{2}>0\right\}$. This concludes the proof.

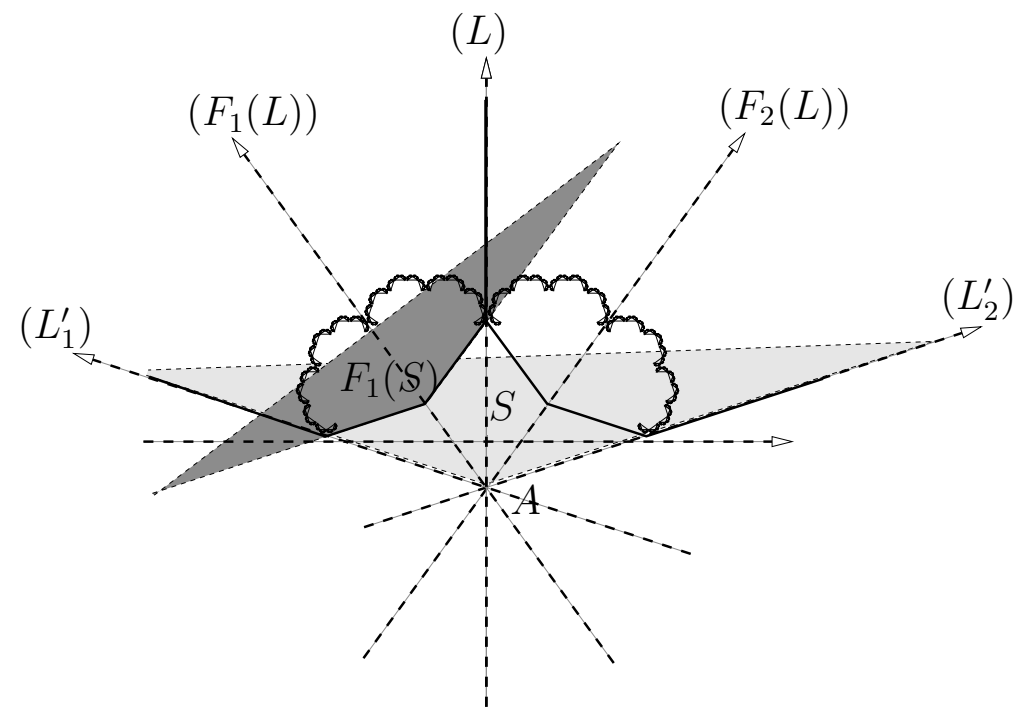

Figure 7: The case $\theta=\pi / 5,(\alpha, \beta)=(a \cos \theta, a \sin \theta)$ with $a=a^{*} \approx 0.56658$.

Remark 11 We have checked numerically that Lemma 9 holds for $0 \leq \theta \leq \pi / 6$, but we have not found a short proof of this. It seems that one needs to iterate the argument above $\kappa-1$ times, where $\kappa$ was introduced in (4), which leads to intricate algebra.

Lemma 10 If $F_{1}$ and $F_{2}$ are given by (1) with $(\alpha, \beta)$ satisfying (5) for $a=a^{*}$ and $\beta \cos \theta-$ $\alpha \sin \theta \geq 0$, then for all $a, 0<a<a^{*}, \Gamma^{\infty}$ is contained in the half-plane $\left\{x: x_{2}>0\right\}$.

Proof. This is an immediate consequence of Proposition 1 and of Lemma 9.

The proof of Theorem 3 in the case $\pi / 6 \leq \theta<\pi / 4$ follows exactly as that of Theorem 2 .

\section{A.3.2 The case $\pi / 4 \leq \theta \leq \pi / 3$}

Lemma 11 Assume that $(\alpha, \beta)$ satisfy (5) for $a=a^{*}$, (6) and (9). Then $\Gamma^{\infty} \cap \tilde{Y}^{0}=\emptyset$ for $a \leq a^{*}$.

Proof. (sketched) Since $\Gamma^{\infty} \subset \overline{F_{1}(S) \cup F_{2}(S)}$ if $a \leq a^{*}$, the desired result will be proved if we show that $\widetilde{Y}^{0} \cap \overline{F_{1}(S)}=\emptyset$ because by symmetry this implies that $\tilde{Y}^{0} \cap \overline{F_{1}(S) \cup F_{2}(S)}=\emptyset$.

The sector $F_{1}(S)$ is delimited by the two lines $F_{1}\left(L_{2}^{\prime}\right)$ and $F_{1}\left(L_{1}^{\prime}\right)$.

The line $F_{1}\left(L_{2}^{\prime}\right)$ is parallel to the vector $(\sin \theta, \cos \theta)$ and contains $F_{1}(A)$. Since $A=(0, \beta-$ $\alpha \cos \theta / \sin \theta) \in \widetilde{Y}^{0}$ from (9), we also have $F_{1}(A) \in F_{1}\left(\widetilde{Y}^{0}\right)$ thus $F_{1}(A) \notin \overline{\widetilde{Y}^{0}}$. It can thus be seen that $\widetilde{Y}_{0}$ is strictly on one side of $F_{1}\left(L_{2}^{\prime}\right)$.

Thus to see that $\widetilde{Y}^{0} \cap \overline{F_{1}(S)}=\emptyset$, we just need to prove that all the vertices of $\widetilde{Y}_{0}$ are strictly contained on one side of $F_{1}\left(L_{1}^{\prime}\right)$. The equation of $F_{1}\left(L_{1}^{\prime}\right)$ is

$$
\cos 3 \theta\left(x_{1}+\alpha-a(\alpha \cos \theta-\beta \sin \theta)\right)+\sin 3 \theta\left(x_{2}-\beta-a\left(-\frac{\alpha}{\sin \theta}+\alpha \sin \theta+\beta \cos \theta\right)\right)=0 .
$$


The desired result will be obtained if injecting $P_{1}=(-1,0)$ and $F_{1}\left(P_{1}\right)=(-\alpha-a \cos \theta, \beta-a \sin \theta)$ in the equation of $F_{1}\left(L_{1}^{\prime}\right)$ yields two negative numbers for all $a, 0 \leq a \leq a^{*}$ : this reads

$$
\begin{aligned}
(\alpha-1) \cos 3 \theta-\beta \sin 3 \theta+2 a \cos \theta(\alpha \cos \theta-\beta \sin \theta) & <0 \\
2(-\beta \sin \theta+\alpha \cos \theta) \cos \theta-\cos 2 \theta & <0 .
\end{aligned}
$$

and these conditions are implied by (9). This concludes the proof.

The proof of Theorem 3 for $\pi / 4 \leq \theta \leq \pi / 3$ then follows as that of Theorem 2, by proving that $\widetilde{Y}^{0} \cap F_{1}(\widetilde{\Omega})=\emptyset$. This comes from the fact that the convex hull of $F_{1}(\widetilde{\Omega})$ is the convex hull of $F_{1}\left(P_{1}\right), F_{1}\left(P_{2}\right)$ and $F_{1}\left(\Gamma^{\infty}\right)$ by Lemma 5 , which, from Lemma 11 , is separated from $\widetilde{Y}^{0}$ by the straight line joining $F_{1}\left(P_{1}\right)$ and $F_{1}\left(P_{2}\right)$.

\section{B Some details in the proof of Theorem 11 for $a=1 / 2$}

The proof of Theorem 11 is similar to that given in the case $a>1 / 2$, except that the set $\widetilde{\Omega}$ and $\widehat{\Omega}$ are defined in a slightly different manner. For the set $\widetilde{\Omega}$, we choose it as in $\S 2.2 .1$ with $\theta=0$ (see Figure 1), so the similitudes are the affine maps given by (12) with $a=1 / 2$. The polygonal cell $\widetilde{Y}^{0}$ is the convex hull of the points $P_{1}=(-1,0), P_{2}=(1,0), F_{2}\left(P_{2}\right)$, and $F_{1}\left(P_{1}\right)$.

Step 1 We limit ourselves to describing the set $\widehat{\Omega}$. It is possible to define a piecewise affine one to one and measure preserving map $H$ from $\widetilde{Y}^{0}$ onto the fractured set

$$
\widehat{Y}^{0}=\text { Interior }\left(((-1,1) \times(0, h)) \backslash\left(\{0\} \times\left(h^{\prime}, h\right)\right)\right),
$$

where $h=\frac{\beta}{2}\left(\alpha+\frac{3}{2}\right)$, and $h^{\prime}$ is a real number smaller than $h$. The map $H$ is such that

- the restriction of $H$ to $\Gamma^{0}$ is the identity,

- for $i=1,2, H$ induces a one to one map from $F_{1}\left(\Gamma^{0}\right)$ onto $[-1,0] \times\{h\}$ and from $F_{2}\left(\Gamma^{0}\right)$ onto $[0,1] \times\{h\}$.

The domains $\widetilde{Y}^{0}$ and $\widehat{Y}^{0}$ are shown in Figure 8 .

In Figure 8, we also show a partition of $\widetilde{Y}^{0}$ into triangles corresponding to a possible map $H$ with $H$ linear on the triangles. The images of the triangles by $H$ are triangles which form a partition of $\widehat{Y}^{0}$. The points on the figure are $I=\left(1+\frac{1}{8}\left(\alpha-\frac{1}{2}\right), \frac{\beta}{8}\right), J=\left(0, \frac{2 \beta}{3}\right), K=$ $\left(1+\frac{7}{8}\left(\alpha-\frac{1}{2}\right), \frac{7 \beta}{8}\right), L=\left(\alpha-\frac{1}{2}, \beta\right), I^{\prime}=\left(1, \frac{\beta}{8}\right), J^{\prime}=\left(0, \frac{2 \beta}{3}\left(1+\frac{1}{8}\left(\alpha-\frac{1}{2}\right)\right)\right), K^{\prime}=\left(1, h-\frac{\beta}{8}\right)$, $M^{\prime}=\left(0, h-\frac{\beta}{8}\left(\alpha-\frac{1}{2}\right)\right)$. In this construction, $h^{\prime}$ is $h^{\prime}=h-\frac{\beta}{8}\left(\alpha-\frac{1}{2}\right)=\beta\left(\frac{3 \alpha}{8}+\frac{13}{16}\right)$.

Let us introduce $\widehat{\mathcal{Q}}^{0}=((-1,1) \times(0, h]) \backslash\left(\{0\} \times\left[h^{\prime}, h\right]\right)$. Let $G_{1}$ and $G_{2}$ be the maps in $\mathbb{R}^{2}$ defined by

$$
G_{1}\left(x_{1}, x_{2}\right)=\left(\frac{1}{2}\left(x_{1}-1\right), h+\frac{x_{2}}{2}\right), \quad G_{2}\left(x_{1}, x_{2}\right)=\left(\frac{1}{2}\left(x_{1}+1\right), h+\frac{x_{2}}{2}\right) .
$$

We define

$$
\widehat{\mathcal{M}}_{\sigma}=G_{\sigma(1)} \circ \cdots \circ G_{\sigma(n)} \quad \text { for } \sigma \in \mathcal{A}_{n}
$$

the sets

$$
\widehat{Y}^{N}=\operatorname{Interior}\left(\widehat{\mathcal{Q}}^{0} \cup \bigcup_{n=1}^{N} \bigcup_{\sigma \in \mathcal{A}_{n}} \widehat{\mathcal{M}}_{\sigma}\left(\widehat{\mathcal{Q}}^{0}\right)\right), \quad \widehat{\Omega}=\operatorname{Interior}\left(\widehat{\mathcal{Q}}^{0} \cup \bigcup_{n=1}^{\infty} \bigcup_{\sigma \in \mathcal{A}_{n}} \widehat{\mathcal{M}}_{\sigma}\left(\widehat{\mathcal{Q}}^{0}\right)\right),
$$




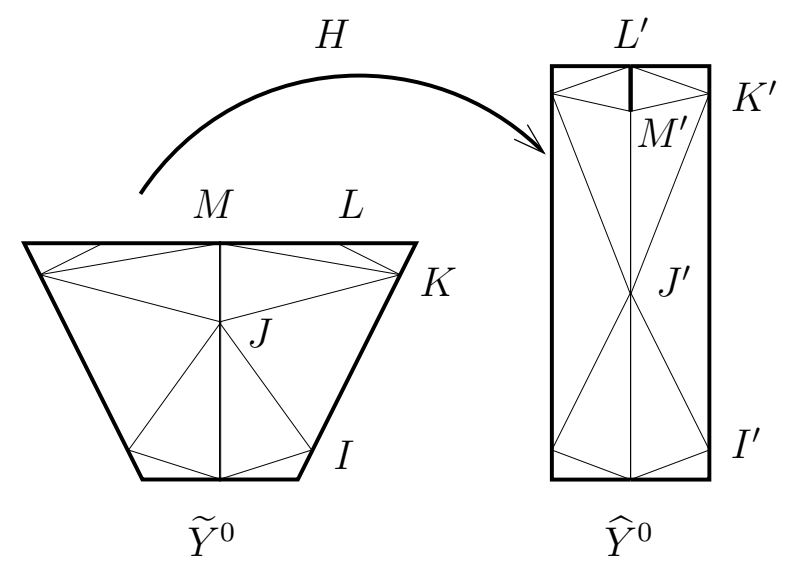

Figure 8: The domains $\tilde{Y}^{0}$ (left) and $\widehat{Y}^{0}$ (right). The restriction of the map $H$ to the triangles covering $\widetilde{Y}^{0}$ is linear.

see Figure 9, and the one to one mapping

$$
\chi^{N}: \mid \begin{aligned}
\widehat{Y}^{N} & \rightarrow \widetilde{Y}^{N}, \\
x & \mapsto \mathcal{M}_{\sigma} \circ H^{-1} \circ \widehat{\mathcal{M}}_{\sigma}^{-1}(x) \quad \text { if } x \in \widehat{\mathcal{M}}_{\sigma}\left(\widehat{Y}^{0}\right) .
\end{aligned}
$$

The set $\widehat{\Omega}$ is obtained by removing an infinite number of vertical segments from the rectangle $(-1,1) \times(0,2 h)$. It is constructed by assembling translated/dilated copies of the set $\widehat{\mathcal{Q}}^{0}$ displayed on the left of Figure 9. Note that $\chi^{N}$ is a piecewise affine function and that the Jacobian of $\chi^{N}$
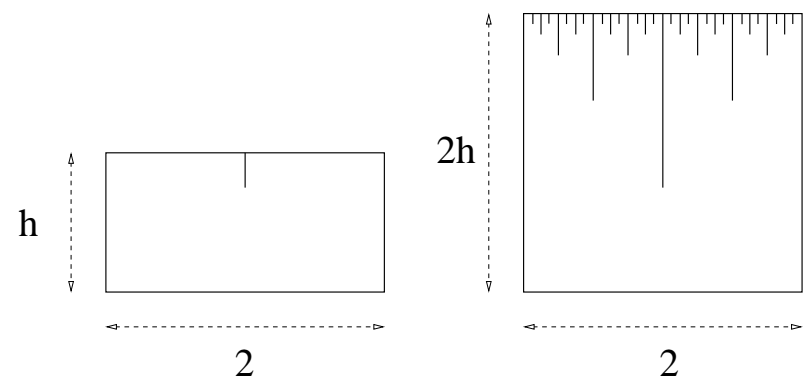

Figure 9: Left: the set $\widehat{\mathcal{Q}}^{0}$. Right: the open set $\widehat{\Omega}$ (only the longest fractures are displayed)

is almost everywhere 1 . Similarly, we can construct a one to one map $\chi^{\infty}$ from $\widehat{\Omega}$ to $\widetilde{\Omega}$, which preserves the measure.

\section{References}

[1] Y. Achdou and N. Tchou. Neumann conditions on fractal boundaries. Asymptotic Analysis, 53(12):61-82, 2007.

[2] Y. Achdou and N. Tchou. Trace theorems on domains with self-similar fractal boundaries. Journal de Mathématiques Pures et Appliquées, 89:596-623, 2008.

[3] G. Berger. Eigenvalue distribution of elliptic operators of second order with Neumann boundary conditions in a snowflake domain. Math. Nachr., 220:11-32, 2000. 
[4] P. G. Ciarlet. The finite element method for elliptic problems. North-Holland Publishing Co., Amsterdam, 1978. Studies in Mathematics and its Applications, Vol. 4.

[5] K. Falconer. Techniques in fractal geometry. John Wiley \& Sons Ltd., Chichester, 1997.

[6] K. J. Falconer. The geometry of fractal sets, volume 85 of Cambridge Tracts in Mathematics. Cambridge University Press, Cambridge, 1986.

[7] J.E. Hutchinson. Fractals and self-similarity. Indiana Univ. Math. J., 30(5):713-747, 1981.

[8] P.W. Jones. Quasiconformal mappings and extendability of functions in Sobolev spaces. Acta Math., $147(1-2): 71-88,1981$.

[9] A. Jonsson. Wavelets on fractals and Besov spaces. J. Fourier Anal. Appl., 4(3):329-340, 1998.

[10] A. Jonsson. Haar wavelets of higher order on fractals and regularity of functions. J. Math. Anal. Appl., 290(1):86-104, 2004.

[11] A. Jonsson and H. Wallin. Function spaces on subsets of $\mathbf{R}^{n}$. Math. Rep., 2(1):xiv+221, 1984.

[12] A. Jonsson and H. Wallin. The dual of Besov spaces on fractals. Studia Math., 112(3):285-300, 1995.

[13] J. Kigami. Analysis on fractals, volume 143 of Cambridge Tracts in Mathematics. Cambridge University Press, Cambridge, 2001.

[14] M.R. Lancia. A transmission problem with a fractal interface. Z. Anal. Anwendungen, 21(1):113-133, 2002.

[15] M.R. Lancia. Second order transmission problems across a fractal surface. Rend. Accad. Naz. Sci. XL Mem. Mat. Appl. (5), 27:191-213, 2003.

[16] B.B. Mandelbrot and M. Frame. The canopy and shortest path in a self-contacting fractal tree. Math. Intelligencer, 21(2):18-27, 1999.

[17] V.G. Maz'ja. Sobolev spaces. Springer Series in Soviet Mathematics. Springer-Verlag, Berlin, 1985. Translated from the Russian by T. O. Shaposhnikova.

[18] P. A. P. Moran. Additive functions of intervals and Hausdorff measure. Proc. Cambridge Philos. Soc., 42:15-23, 1946.

[19] U. Mosco. Dirichlet forms and self-similarity. In New directions in Dirichlet forms, volume 8 of AMS/IP Stud. Adv. Math., pages 117-155. Amer. Math. Soc., Providence, RI, 1998.

[20] U. Mosco. Energy functionals on certain fractal structures. J. Convex Anal., 9(2):581-600, 2002. Special issue on optimization (Montpellier, 2000).

[21] U. Mosco and M. A. Vivaldi. Variational problems with fractal layers. Rend. Accad. Naz. Sci. XL Mem. Mat. Appl. (5), 27:237-251, 2003.

[22] R. S. Strichartz. Function spaces on fractals. J. Funct. Anal., 198(1):43-83, 2003.

[23] R.S. Strichartz. Analysis on fractals. Notices Amer. Math. Soc., 46(10):1199-1208, 1999.

[24] H. Triebel. Fractals and spectra, volume 91 of Monographs in Mathematics. Birkhäuser Verlag, Basel, 1997. Related to Fourier analysis and function spaces.

[25] S. K. Vodop'janov, V. M. Gol'dšteřn, and T. G. Latfullin. A criterion for the extension of functions of the class $L_{2}^{1}$ from unbounded plane domains. Sibirsk. Mat. Zh., 20(2):416-419, 464, 1979. 